\title{
SMALL-ANGLE AND SURFACE SCATTERING FROM POROUS AND FRACTAL MATERIALS
}

S. K. SNHA
Advanced Photon Source
Argonne National Laboratory
Argonne, IL 60439, USA

\begin{abstract}
We review the basic theoretical methods used to treat small-angle scattering from porous materials, treated as general two-phase systems, and also the basic experimental techniques for carrying out such experiments. We discuss the special forms of the scattering when the materials exhibit mass or surface fractal behavior, and review the results of recent experiments on several types of porous media, and also SANS experiments probing the phase behavior of binary fluid mixtures or polymer solutions confined in porous materials. Finally, we discuss the analogous technique of off-specular scattering from surfaces and interfaces which is used to study surface roughness of various kinds.
\end{abstract}

\section{Introduction}

Small-angle X-ray scattering (SAXS) and small-angle neutron scattering (SANS) are by now well-established techniques to study the structure of materials on lengthscales of $20 \AA-10 \mu$. We shall not attempt to cover the important applications of these techniques in the field of biology, polymer science or metallurgy, but concentrate on studies of microporous materials, fractally aggregated objects, etc. While these techniques provide indirect information about the sample, which in general must be fitted by models, they have the advantage of providing in-situ global statistical data on the sample and are not sensitive to sample environments.

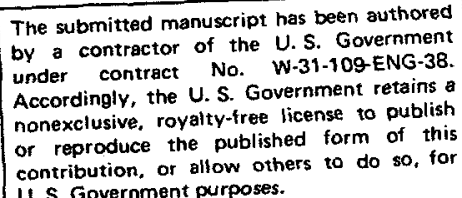




\section{DISCLAIMER}

This report was prepared as an account of work sponsored by an agency of the United States Government. Neither the United States Government nor any agency thereof, nor any of their employees, make any warranty, express or implied, or assumes any legal liability or responsibility for the accuracy, completeness, or usefulness of any information, apparatus, product, or process disclosed, or represents that its use would not infringe privately owned rights. Reference herein to any specific commercial product, process, or service by trade name, trademark, manufacturer, or otherwise does not necessarily constitute or imply its endorsement, recommendation, or favoring by the United States Government or any agency thereof. The views and opinions of authors expressed herein do not necessarily state or reflect those of the United States Government or any agency thereof. 


\section{DISCLAIMER}

Portions of this document may be illegible in electronic image products. Images are produced from the best available original document. 
A particular advantage of SANS as opposed to SAXS is the ability to contrastmatch selected portions of the sample and this has been explored in many areas as we shall see.

A somewhat related technique is that of diffuse or off-specular scattering from surfaces and interfaces which provides information about such systems analogous to the information small-angle scattering provides about bulk systems, and this will be discussed at the end of this lecture.

The basic methodology of small-angle scattering has been discussed in a variety of excellent reviews, ${ }^{\text {th }}$ and we shall simply review that part of the formalism which is relevant to the study of porous materials. Neglecting any inelasticity in the scattering , the number of particles per second scattered by a sample into a detector is given by

$$
I=S(Q)\left(I_{0} / A\right)(\Delta \Omega)
$$

where $I_{0}$ is the number of particles per second in the incident beam of cross-sectional area $A,(\Delta \Omega)$ is the solid angle subtended at the sample by the detector, $S(Q)$ is the scattering function characterizing the sample and $\mathbf{Q}$ is the so-called wavevector transfer defined by

$$
\mathbf{Q}=\mathbf{k}_{\mathbf{1}}-\mathbf{k}_{\mathbf{f}}
$$

where $k_{i}, k_{f}$ are the wavevectors of the incident and scattered radiation respectively. The magnitude of $Q$ is given by $Q=2 k_{0} \operatorname{Sin} \vartheta$ where $2 \vartheta$ is the angle of scattering, where $k_{0}=2 \pi / \lambda, \lambda$ being the wavelength of the incident radiation. In general the small angle regime is defined by $Q<<k_{0} . S(Q)$ in general is a function of the average of the instantaneous positions of all the particles in the scattering system, but in the small-angle regime (defined roughly by the range $0<$ $Q<0.3 \AA^{-1}$ ) we may ignore the atomic and molecular structure of the constituents and deal only with the spatial variations (on length scales from a few $\mathrm{nm}$ on up) of 
the scattering length density (SLD) $\rho(\mathbf{r})$ of the sample. For SANS experiments, $\rho(\mathbf{r})$ is defined by

$$
\rho(\mathbf{r})=\sum_{i} b_{i} n_{i}(\mathbf{r})
$$

where $b_{i}$ is the nuclear scattering length ${ }^{s}$ of nucleus of type $i$, and $n_{i}(r)$ is the associated number density of such nuclei, while for SAXS experiments, $\rho(\mathbf{r})$ is defined by

$$
\rho(\mathbf{r})=\left(\mathrm{e}^{2} / \mathrm{mc}^{2}\right) \mathrm{n}_{\mathrm{el}}(\mathbf{r})
$$

where the factor $\left(\mathrm{e}^{2} / \mathrm{mc}^{2}\right)$ is the Thompson scattering length of the electron, and $\mathrm{n}_{\mathrm{el}}(\mathbf{r})$ is the electron number density. Since a uniform scattering length density does not scatter radiation (except in the forward direction), $S(Q)$ will depend only on the deviations of $\rho(\mathbf{r})$ about its mean, or what is referred to as the contrast. The Kinematic or Born approximation to the scattering [6], where multiple scattering effects are neglected, then yields for the scattering function $S(Q)$ the expression

$$
S(\mathbf{Q})=\iint \mathrm{d} \mathbf{r} d \mathbf{r}^{\prime}\left\langle\delta \rho(\mathbf{r}) \delta \rho\left(\mathbf{r}^{\prime}\right)\right\rangle e^{\mathrm{i} \mathbf{Q}\left(\mathbf{r}^{\prime}-\mathbf{r}\right)} \equiv \iint \mathrm{d} \mathbf{r} \mathrm{d} \mathbf{r}^{\prime} \gamma\left(\mathbf{r}-\mathbf{r}^{\prime}\right) \mathrm{e}^{\mathrm{i} \mathbf{Q}\left(\mathbf{r}^{\prime}-\mathbf{r}\right)}(5)
$$

where the statistical average is taken over the whole system. (This statement has to be modified if the incident radiation is highly coherent, as discussed at the end of this lecture.) It is commonly assumed that such an average depends only on the magnitude of $\mathbf{R} \equiv \mathbf{r}-\mathbf{r}^{\prime}$, for an isotropic and translationally invariant random porous medium. In Eq. (5), $\delta p(\mathbf{r})$ is defined as the fluctuation from the average SLD as explained above. If we are dealing with a particulate system (or a system of random cavities) and these are far enough apart that the interference effects between the scattering from different particles (which will occur at values of $Q$ typically of order $(2 \pi / d)$ where $d$ is the average interparticle distance) are not important in the range of $Q$ studied in the experiment, then Eq.(5) simplifies to

$$
S(\mathbf{Q})=(\Delta \rho)^{2} \sum_{i} v_{i}^{2} f_{i}^{2}(\mathbf{Q})
$$


where the sum is over all particles, $v_{i}$ is the volume of the $i^{\text {th }}$ particle, $\Delta \rho$ is its SLD contrast with the average medium (assumed uniform throughout the particle and the same for all particles) and $\mathrm{f}_{i}$ is its form factor defined by

$$
f_{i}(Q)=\frac{1}{v_{i}} \int_{v_{i}} d r e^{-i Q \cdot r}
$$

where the integral is over the particle volume. In general, one can make a spherical average of $f_{i}^{2}(Q)$ and assume some law of polydispersity in size of the particles to carry out the weighted sum in Eq.(6). In such a restrictive case (dilute system of random particles), by making an expansion for small $\mathbf{Q}$, one finds that

$$
S(Q)=S(0)-\frac{1}{3} R_{g}^{2} Q^{2}+\ldots
$$

where

$$
R_{g}^{2}=\sum_{i}\left(1 /_{v_{i}}\right) \int_{v_{i}} d r \cdot r^{2}
$$

is the average radius of gyration of the particles. Eq.(8) suggests (at least for small Q) in this case the approximation

$$
S(Q)=S(0) \exp \left(-\frac{1}{3} R_{g}^{2} Q^{2}\right)
$$

which is the famous Guinier approximation ${ }^{1}$ for scaling from a dilute system of uniform particles. As we shall see in Section 2, this is of limited usefulness in the case of a real random porous medium.

\section{Scattering from Porous Media}

Contrary to what one might think initially, it is rare to find a porous material that consists of tiny isolated voids randomly embedded in a solid material. For such a simple (but unrealistic) case, one may employ Babinet's principle and use the formula for scattering from a collection of particles and include polydispersity to obtain the dependence of $S(Q)$ on the pore size distribution as discussed in the 
previous section. A more realistic representation for a porous solid is in terms of a two-phase random or bicontinuous medium (see Fig. 1) where one phase is simply the pore space and the other the solid phase, assumed to be of uniform scattering length density at least for length scales $>1 / \mathrm{Q}_{\max }$ Since we can imagine the pore space being filled with liquid in some cases, let us generally assume the SLD in these two phases to be $\rho_{1}$ and $\rho_{2}$, respectively. Following Ref.[7], let the volume fractions of the two phases be $\phi_{1}$ and $\phi_{2}$, respectively, $\left(\phi_{1}+\phi_{2}=1\right)$. The average SLD of the system is given by

$$
\bar{\rho}=\phi_{1} \rho_{1}+\phi_{2} \rho_{2}
$$

The effective SLD contrasts for phase 1 and phase 2 are given by

$$
\begin{aligned}
& \eta_{1}=\rho_{1}-\bar{\rho}=\phi_{2}\left(\rho_{1}-\rho_{2}\right)=\phi_{2} \Delta \rho \\
& \eta_{2}=\rho_{2}-\bar{\rho}=-\phi_{1}\left(\rho_{1}-\rho_{2}\right)=-\phi_{1} \Delta \rho
\end{aligned}
$$

Let us pick at random two points $(A, B)$ in the system separated by a distance $R$. Let $P_{11}(R)$ be the conditional probability that if point $A$ is in phase 1 , then point $B$ is also in phase 1. Similarly for $P_{12}(R), P_{21}(R)$, and $P_{22}(R)$. Obviously, by definition

$$
\begin{array}{ll} 
& \mathrm{P}_{11}(\mathbf{R})+\mathrm{P}_{12}(\mathbf{R})=1 \\
& \mathrm{P}_{21}(\mathbf{R})+\mathrm{P}_{22}(\mathbf{R})=1
\end{array}
$$

The absolute probability that both points are in phase 1 may be called $\tilde{\mathrm{P}}_{11}(\mathbf{R})$ and must be given by

$$
\tilde{\mathbf{P}}_{11}(\mathbf{R})=\phi_{1} P_{11}(\mathbf{R})
$$

Similarly

$$
\tilde{\mathrm{P}}_{12}(\mathbf{R})=\phi_{1} \mathrm{P}_{12}(\mathbf{R})=\tilde{\mathrm{P}}_{21}(\mathbf{R})=\phi_{2} \mathrm{P}_{21}(\mathbf{R}) \text { (by symmetry) }
$$

and

$$
\tilde{\mathrm{P}}_{22}(\mathbf{R})=\phi_{2} \mathrm{P}_{22}(\mathbf{R})
$$

Let us define a function $\gamma_{0}(\mathbf{R})$ such that

$$
P_{11}(R)=\phi_{1}+\phi_{2} \gamma_{0}(R)
$$


Then by Eq.(15)

$$
P_{12}(\mathbf{R})=\phi_{2}-\phi_{2} \gamma_{0}(\mathbf{R})
$$

By Eq.(17)

$$
P_{21}(\mathbf{R})=\phi_{1}-\phi_{1} \gamma_{0}(\mathbf{R})
$$

and again by Eqs.(18)\&(15)

$$
\mathrm{P}_{22}(\mathbf{R})=\phi_{2}+\phi_{1} \gamma_{0}(\mathbf{R})
$$

From the above arguments, we have from Eq. (5),

$$
\begin{aligned}
& \gamma(\mathbf{R}) \equiv\left\langle\delta \rho(\mathbf{r}) \delta \rho\left(\mathbf{r}^{\prime}\right)\right\rangle=\langle\delta \rho(0) \delta \rho(\mathbf{R})\rangle \\
& =\eta_{1}^{2} \tilde{\mathrm{P}}_{11}(\mathbf{R})+2 \eta_{1} \eta_{2} \tilde{\mathrm{P}}_{12}(\mathbf{R})+\eta_{2}^{2} \tilde{\mathrm{P}}_{22}(\mathbf{R})
\end{aligned}
$$

where $\mathbf{R}=\mathbf{r}^{\prime}-\mathbf{r}$ and we have assumed overall statistical translational invariance. By Eqs. (14)-(21), we thus have after some algebra,

$$
\gamma(\mathbf{R})=(\Delta \rho)^{2} \phi_{1} \phi_{2} \gamma_{0}(\mathbf{R})
$$

so that

$$
S(\mathbf{Q})=\mathrm{V}(\Delta \rho)^{2} \phi_{1} \phi_{2} \int \mathrm{d} \mathbf{R} \gamma_{0}(\mathbf{R}) \mathrm{e}^{-\mathrm{iQ} \cdot \mathbf{R}}
$$

where $V$ is the sample volume.

Assuming $\gamma_{0}(\mathbf{R})$ to be isotropic, Eq.(23) may be simplified to

$$
S(Q)=V(\Delta \rho)^{2} \phi_{1} \phi_{2} \int_{0}^{\infty} d R 4 \pi R^{2} \gamma_{0}(R)\left(\frac{\sin Q R}{Q R}\right)
$$

Note that, by definition, $P_{11}(0)=P_{22}(0)=1$ and $P_{12}(0)=P_{21}(0)=0$ which by Eqs. (17)-(20) implies that $\gamma_{0}(0)=1$. Note also that by Eq. (24),

$$
S(0)=V(\Delta \rho)^{2} \phi_{1} \phi_{2} v_{c}
$$

where

$$
v_{c}=\int_{0}^{\infty} \mathrm{dR} 4 \pi R^{2} \gamma_{0}(R)
$$

and is called the comelation volume. If this volume is larger than the finite coherence volume of the radiation (typical neutron coherence lengths being $\sim$ a w hundred angstroms at best for such experiments) then the integral in Eq.(26) is reduced to being only over the radiation coherence volume. The inverse Fourier 
transform of Eq.(24) yields a way of obtaining $\gamma_{0}(R)$ directly from the measured $S(Q):$

$$
\gamma_{0}(R)=\frac{1}{2 \pi^{2} V(\Delta \rho)^{2} \phi_{1} \phi_{2}} \int_{0}^{\infty} d_{q q}{ }^{2} S(Q)\left(\frac{\sin Q R}{Q R}\right)
$$

Using the relation $\gamma_{0}(0)=1$, we obtain

$$
\mathrm{Q}_{p} \equiv \int_{0}^{\infty} \mathrm{dQQ} \mathrm{Q}^{2} \mathrm{~S}(\mathrm{Q})=2 \pi^{2} \mathrm{~V}(\Delta \rho)^{2} \phi_{1} \phi_{2}
$$

where $Q_{p}$ (proportional to the integral of $S(Q)$ over all $Q$ ) is called the Porod invariant [2] and does not depend on the detailed morphology of the pore structure.

The correlation volume can be obtained from $S(O)$ if it can be reliably extrapolated from finite $Q$ values and $Q_{p}$ can be obtained from Eq. (28). Let us now consider the small $\mathrm{R}$ expansion of $\gamma_{0}(R)$. It can be proved that for smooth internal surfaces,

$$
\gamma_{0}(R)=1-R / \bar{\ell}+\cdots
$$

where

$$
\frac{1}{\bar{\ell}}=\frac{1}{4 \phi_{1} \phi_{2}}(\mathrm{~S} / \mathrm{V})
$$

where $\bar{\ell}$ is a mean chord length (averaged across the pores and the solid), and $S$ is the internal surface area of the pores. Then, substituting in Eq.(24) for $S(Q)$, integrating by parts and keeping the leading term as $Q \rightarrow \infty$ (for this purpose we neglect oscillatory terms in the integral, which average out except for perfectly regular systems), we obtain the large $Q\left(Q \gg \frac{1}{\bar{\ell}}\right)$ behavior of $S(Q)$

$$
S(Q) \approx 2 \pi(\Delta \rho)^{2} S / Q^{4}
$$

which is the famous Porod's Law ${ }^{2}$ for the asymptotic form of the scattering at large $\mathrm{Q}$ (See Fig. 2a). A useful relation, using Eqs. (31) and (28) is

$$
S / V=\pi \phi_{1} \phi_{2} / Q_{p} \lim _{Q \rightarrow \infty}\left(Q^{4} S(Q)\right)
$$


Thus the internal surface-to-volume ratio for a porous material can be found from scattering experiments alone (if $\Phi_{1}$ and $\Phi_{2}$ are known) and can be checked for consistency against separate measurements of the internal surface area and porosity (e.g., from vapor pressure isotherm and porosimetry measurements). Porod's Law is not always obeyed in the sense that, for many porous materials, asymptotic power laws for $S(Q) \sim Q^{-v}$ have been observed, where $v$ varies between 3 and 4 (see Fig. 2b). This can be explained in terms of a rough internal surface, which at these length scales (of order $Q^{-1}$ ) behaves like a self-similar fractal surface. This will be discussed in the next section. For such surfaces, $S / V$ changes with $1 / Q$ in Eq. (34), reflecting the fact that for a fractal surface the area depends on the length scale chosen.

Turning now to specific models for $\gamma_{0}(R)$, one of the best-known expressions is due to Debye, Anderson, and Brumberger[7], who basically used a statistical model for the porous medium so that $\gamma_{0}(R)$ was given by the expression resulting from Poisson statistics:

$$
\gamma_{0}(R)=e^{-R / a}
$$

where $a$ is a length that can be taken as characterizing either the average pore size or grain size (for a porous medium consisting of compacted grains). This results in an expression for $S(Q)$ given by

$$
S(Q)=8 \pi V(\Delta \rho)^{2} \phi_{1} \phi_{2} a^{3} /\left(1+Q^{2} a^{2}\right)^{2}
$$

which has the asymptotic form of Porod's Law (Eq.(31) and is equivalent to putting $\bar{\ell}=\mathrm{a}$ in Eqs. (29) and (30).

Fig. 2(a) shows small-angle x-ray scattering from a series of silica gels, ${ }^{8}$ which are porous materials produced by sintering aggregations of silica particles, as exhibited in the micrograph in Fig.3. The curves show superpositions of $S(Q)$ for several such gels (with appropriate scaling for each of the axes) and are seen to collapse on a single curve, showing that scattering from each member of this family 
of gels has the same form, except for a single adjustable length scale, the size of the "building block particles". This length scale is obtained by fitting the intermediate to large $Q$ parts of $S(Q)$ to the form of Eq. (34). (Porod's Law is obeyed quite well here, indicating relatively smooth internal surfaces.) At small $Q$, the $S(Q)$ rises according to a different power law, which is related to a mass-fractal structure of inhomogeneities existing at large length scales (discussed later).

Vycor glass is extremely popular as a "model porous medium" for a variety of experiments. It is produced by spinodal decomposition on quenching a borosilicate melt from high temperature, resulting in a phase separation of a boron-rich phase and a silica-rich phase. The former is then removed by acid leaching leaving the pore space, and the material resembles that shown in Fig.4, i.e., a bicontinuous 2phase system resulting from the late stages of spinodal decomposition. As might be expected, a characteristic quasi-periodicity exists in this structure (the distribution of distances from an internal surface into a pore through the solid and to the next pore surface is peaked around a characteristic length of $250 \AA$, the average pore diameter being $80 \AA$ and the porosity being typically $28 \%$ ), and $S(Q)$ for Vycor $^{9-11}$ shows a peak as illustrated in Fig. $2 b$ (as measured by SANS). Note that the asymptotic slope (on a $\log -\log$ plot of $S(Q)$ ) shows an exponent of 3.5 instead of the Porod exponent of 4 , indicating a fractally rough surface at small length scales, as will be discussed in the next section. (However, this deviation may also be due to compositional inhomogeneities at the interface.)

A simple and intuitively appealing method of characterizing the morphology of a two-phase porous medium is in terms of chord distributions across each of the two phases (e.g. solid-occupied space and pore space) as indicated schematically in Fig. 1, i.e. the probability distribution of lengths along a particular direction between one interface and the next. (For an isotropic porous medium, these distributions will obviously be independent of the direction chosen.) There are obvious similarities to the definition of the Debye function $\gamma_{0}(\mathbf{R})$. It has not proved 
possible, however, to rigorously express $S(Q)$ in terms of such chord distributions, without further restrictive assumptions. For a random bicontinuous porous medium, the most obvious assumption which suggests itself is that the probabilities of intersecting successive solid-pore and pore-solid interfaces depend only on the chord distributions and are otherwise totally uncorrelated with previous or successive intersections. It is then possible to devise a formal expression for $\gamma_{0}(\mathbf{R})^{12}$, but writing down an explicit closed-form expression for $S(Q)$ is difficult except in special cases. An explicit form for $S(Q)$ was devised by Lin and Sinha ${ }^{13}$ using a simple phenomenological model similar in spirit to that of Mering and Tchoubar ${ }^{12}$, but with more restrictive assumptions.

A more sophisticated model has been given by Berk ${ }^{14}$ starting from the socalled Cahn construction for an internal surface, resulting from choosing contours of the function

$$
F(\mathbf{r})=\frac{1}{\sqrt{N}} \sum_{i} \cos \left(\mathbf{k}_{i} \cdot \mathbf{r}+\phi_{i}\right)
$$

where $\mathbf{k}_{i}$ are a set of $\mathrm{N}$ vectors random in direction but having a narrow distribution in length, and $\phi_{i}$ are a set of random phases. One then chooses a cutoff value for this function, so that all points for which $F(r)$ is smaller than this cut off can be called "pore space" and all points for which $F(\mathbf{r})$ is greater can be called "solid". For the special case of so-called isometric topology, where this cut-off has the value zero and $\phi_{1}=\phi_{2}$, Berk proves the remarkably elegant result

$$
\gamma_{0}(R)=(2 / \pi) \arcsin \left[\mathrm{j}_{0}(\mathrm{kR})\right]
$$

while for the general case, he derives an expression for $\gamma_{0}(R)$ in terms of a powerseries expansion of $j_{0}(k R)$. Here $j_{0}$ is the spherical Bessel function, and $k$ is the magnitude of the wavevectors $k_{i}$. In practice, one averages $j_{0}(k R)$ over a narrow distribution of $\mathrm{k}$ centered about some mean value. Using this formalism, Berk has calculated $S(Q)$ functions which remarkably resemble the scattering from spinodally decomposed systems, such as Vycor, as well as bicontinuous microemulsion 
phases. ${ }^{14}$ These show the characteristic peak at finite $Q$ seen in the scattering from such systems.

Another analytic form for $S(Q)$ for such systems has been given by Teubner and Strey ${ }^{15}$ based on Ginsburg-Landau free energy theory and may be written as

$$
S(Q)=V(8 \pi / \zeta)(\Delta \rho)^{2} C_{2} /\left(a_{1}+C_{1} Q^{2}+C_{2} Q^{4}\right)
$$

where $\zeta$ is the correlation length and $\mathrm{a}_{1}, \mathrm{C}_{1}, \mathrm{C}_{2}$ are constants. This corresponds to a Debye correlation function

$$
\gamma_{0}(R)=\frac{d}{2 \pi R} e^{-R / \zeta} \sin (2 \pi R / d)
$$

where $d$ is a typical domain size. The form of $\gamma_{0}(R)$ reflects the quasi-periodicity of the medium, and Eq. (37) has been successfully used to fit bicontinuous microemulsion data. It possesses the characteristic peak (as seen in Vycor, for instance).

Chen et $\mathrm{al}^{16}$ have introduced a modification of Berk's model which borrows, some concepts also from the Teubner-Strey model. Their $\gamma_{0}(R)$ is obtained from an expression similar to Eq. (36) but with jo(kR) (which in Berk's model is really the two-point autocorrelation function of $F(r)$ in Eq. (35)) replaced by the Fourier transform of an inverse sixth-order polynomial in $k$ which contains three parameters $a, b, c$. These authors also derive an expression for the average Gaussian curvature of the interface in terms of these parameters, and are thus able to obtain values for this curvature by fitting $S(Q)$ for an isometric bicontinuous microemulsion. ${ }^{16}$

Another model for a spinodally decomposed system at late times has been developed by $\mathrm{Li}$ and Ross. ${ }^{17}$ These authors use arguments to show that if magnitudes of allowed wavevectors have a very narrow range centered on $k_{0}$, then $\mathbf{S}(\mathbf{Q})$ may be written as 


$$
S(Q)=C\left\langle F^{2}(Q)\right) \frac{1}{Q k_{0}}\left\{\zeta /\left[1+\left(Q-k_{0}\right)^{2} \zeta^{2}\right]-\zeta /\left[1+\left(Q+k_{0}\right)^{2} \zeta^{2}\right]\right\}
$$

where $C$ is a constant, $F(Q)$ is a "pore form factor" and $\zeta$ represents a correlation length for the decay of the sinusoidal concentration fluctuations associated with the spinodal decomposition. Using this form, $\mathrm{Li}$ and Ross were able to get a remarkably good fit to the scattering from dry Vycor glass. ${ }^{17}$

It is to be noted that since all these forms are based on smooth internal surfaces, they cannot reproduce anything except an asymptotic $Q^{-4}$ behavior for $S(Q)$, although the model of Ref. [13] gives a prescription for modifying the $S(Q)$ in the case of roughness.

\section{Scattering from Fractal Systems}

We next consider porous solids produced by the rather tenuous aggregation of small or colloidal particles, which often have local mass-fractal structure. Many types of silica gels, xerogels, and aerogels are of this type. Scattering experiments can often provide valuable clues regarding the process of formation of such porous solids. These materials can have extremely high (up to $\sim 98 \%$ ) porosity and still stay amazingly rigid. For such systems Eq. (5) may be written as

$$
S(\mathbf{Q})=N f^{2}(Q)(\Delta \rho)^{2} \int d \mathbf{R} g(\mathbf{R}) \mathrm{e}^{-\mathrm{iQ} \cdot \mathbf{R}}
$$

where $\mathrm{N}$ is the total number of particles, $\mathrm{f}(\mathrm{Q})$ is the average form-factor (or Fourier transform of the shape function of the individual particles), $(\Delta \rho)$ is the contrast between the SLD of the particle and that of the embedding medium (usually vacuum or some solvent), and $g(\mathbf{R})$ is the pair-distribution function for the centers of the particles, i.e., the probability per unit volume that given a particle center at the origin, another center can be found at a distance $\mathbf{R}$. This is a function of the 
magnitude $\mathrm{R}$ only, and for a fractal object can be obtained from the definition that within a radius $R$ there are on the average $C R^{D}$ particles (where $C$ is a constant and $D$ is the mass fractal dimension). ${ }^{18}$ By differentiating, we obtain

$$
\begin{array}{ll} 
& 4 \pi R^{2} g(R) d R=D C R^{D-1} d R \\
\text { or } \quad & g(R)-R^{D-3}
\end{array}
$$

In general $O<D<3$, so that the $3 D$ Fourier transform of $g(R)$ yields an $S(Q)$ which goes as $Q^{-D}$. However, fractal correlations exist only up to a finite length scale (a typical cluster size) and may also not be obeyed at short length scales, e.g., nearest neighbor distances.

Neglecting the latter effect (which affects only the large $Q$ behavior of $S(Q)$ ), we can take finite cluster sizes into account by choosing instead of Eq. (42), a modified form for $g(R)$, i.e. ${ }^{19.20}$

$$
g(R)=\delta(R)+A R^{D-3} e^{-R / \xi}+C^{\prime}
$$

where the delta-function at the origin is due to the particle correlation "with itself", $A$ and $C^{\prime}$ are constants and $\xi$ represents the correlation length of the fractal. This expression may be Fourier transformed to yield for Eq. (40) the expression (neglecting the forward scattering at $Q=0$ ),

$$
S(Q)=N f^{2}(Q) \Delta \rho^{2}\left[1+\frac{A}{\left(1+Q^{2} \xi^{2}\right)^{D / 2}} \frac{\left[1+Q^{2} \xi^{2}\right]^{\frac{1}{2}}}{Q \xi} \frac{\sin \left[(D-1) \tan ^{-1}(Q \xi)\right]}{D-1}\right]
$$

As expected, provided $Q$ is much less than the inverse of the particle size $a, S(Q)$ behaves like $Q^{-D}$ for $Q \gg>\xi^{-1}$ and saturates when $Q \sim \xi^{-1}$. For $Q^{-1}$ much less than the particle size, $S(Q)$ behaves like the square of the single particle form factor $f^{2}(Q)$ and 
eventually rolls off as $Q^{-4}$ if the particles are smooth or as $Q^{-v}(3<v<4)$ if the particles are fractally rough. ${ }^{21}$ The above expression has been successfully used to fit the scattering from a variety of fractal systems and to study the dependence of $D$, and $\xi$ on porosity. Fig. 5 shows $S(Q)$ for a series of acid-catalyzed aerogels by Frisken et al. ${ }^{22}$ fitted to the above expression and indicating the corresponding values of the parameters $D, \xi$. It may be seen that there is a slight tendency for both $\mathrm{D}$ and $\xi$ to increase as the $\mathrm{pH}$ of the solution from which these samples were grown decreases, at constant porosity. By studying silica gels in situ during the formation process, the evolution of $\xi$ with time as the fractal clusters grow and saturate has also been studied. ${ }^{23}$

As discussed in the previous section, the asymptotic form at large $Q$ of $S(Q)$ for many porous solids in practice does not obey Porod's Law $\left(Q^{-1}\right)$ but rather $Q^{-v}$ where $v$ is an exponent between 3 and 4. This was first explained by Bale and Schmidt ${ }^{21}$ in terms of an internal surface which is not smooth (as assumed by the Porod theory) but is actually a self-similar fractal surface." From the "tiling" definition of such a surface ${ }^{18}$ these authors were able to show that the small $R$ expansion of $\gamma_{0}(R)$ in Eq. (29) should be replaced by

$$
\gamma_{0}(R)=1-C^{3-D_{1}}+\cdots
$$

where $D_{3}$ is the surface fractal dimension of the internal surface, and $C$ is a constant. Wong and Bray ${ }^{24}$ have pointed out that Eq. (45) needs to be considered more carefully as it gives completely the wrong result as $D_{3} \rightarrow 3$, predicting vanishing small angle scattering. In fact they showed that the factor $C$ contains $\left(3-D_{3}\right)$ in the denominator, and deduced for $S(Q)$ in the asymptotically large $Q$ limit, 


$$
S(Q)=\frac{\pi(\Delta \rho)^{2} S_{2} \ell_{2}{ }^{D_{s}-2} \Gamma\left(5-D_{s}\right) \sin \left[\pi\left(3-D_{s}\right) / 2\right]}{\left(3-D_{s}\right) Q^{6-D_{s}}}
$$

which must replace Eq. (31). In this expression $S_{2}$ is the "smooth" surface area, measured at the length scale $l_{2}$ where the surface fractal behavior cuts off, and $\Gamma$ is the gamma function. Eq. (46) yields finite scattering as $D_{3} \rightarrow 3$, as it should. Measurements on porous media by Hurd et al. ${ }^{25}$ have found that inclusion of the (3$D_{8}$ ) factor in the prefactor to Eq. (46) yields the correct BET surface area from scattering data.

Note that for a smooth surface, $D_{3}=2$ and Porod's Law is recovered. In general $2 \leq D_{s} \leq 3$ so that measurement of the exponent $v$ can be used to obtain the surface fractal dimension. Schmidt and co-workers have shown that a variety of porous systems particularly coals and charcoals ${ }^{2126}$ have fractal dimensions greater than 2 . Wong et al. ${ }^{27}$ have shown, in a series of SANS experiments on various rocks, that these also have surface fractal dimensions larger than 2 (and often close to 3). Most measured samples of Vycor ${ }^{9-11}$ also exhibit a value of the exponent $v$ close to 3.5 , indicating a value of $D_{3}$ of $\sim 2.5$.

Self-similar fractal surfaces are however only one possible kind of rough surface. Another common form of roughness (typically seen in films and other surfaces) is self-affine roughness, defined by ${ }^{20.29}$

$$
g(R) \equiv\left\langle[\delta z(R)-\delta z(0)]^{2}\right\rangle=C R^{2 h}
$$

where $h$ is the self-affine roughness exponent and has the range $0<h<1$ and $\delta z(R)$ is the height fluctuation above the average surface at lateral position $\mathbf{R}$. The rootmean-square height deviation $[g(R)]^{\frac{1}{2}}$ always becomes much less than $R$ for large enough $\mathrm{R}$ so that such surfaces look flat at large enough length scales, but at small 
length scales $\left(R \leq C^{1 / 2(1-h)}\right)$ such surfaces approach self-similar fractal surfaces. Their surface fractal dimension $D_{s}$ is related to $h$ by

$$
\mathrm{D}_{\mathrm{s}}=3-\mathrm{h}
$$

Wong ${ }^{29}$ first showed that the asymptotic form of $S(Q)$ for an isotropic average of such surfaces had the form

$$
S(Q)-A / Q^{4}+B / Q^{3+h}
$$

The first term is Porod's Law arising from the larger length scale (smooth) behavior, while from Eq. (48) the second term is seen to be identical to the Bale-Schmidt form in Eq. (46). Eq. (49) was also derived by Sinha et al..$^{28}$ using a different method. Clearly the second term will dominate the asymptotic behavior at large enough $Q$, although the $Q^{-4}$ term may get mixed up with other behavior at intermediate $Q$ and thus it is often difficult to decide whether an internal surface is self-similarly or self-affinely rough.

In any case, we have the general behavior for $S(Q)$ for a large class of porous materials produced by aggregation (and sintering) of colloidal or granular particles first sketched by Schaeffer and co-workers ${ }^{25}$ and shown schematically in Fig.6. At large $Q$, where we are sensitive only to the shortest length scales (i.e. the internal surfaces) we see power-law behavior characteristic of surface fractals (with Porod's Law being the limiting case of $\left.D_{3}=2\right)$ and at intermediate $Q\left(Q^{-1} \geq\right.$ the particle size), we cross over to the power-law characteristic of the mass fractal behavior of the particle aggregates, while at small $Q\left(Q^{-1} \geq\right.$ aggregate cluster size), $S(Q)$ saturates.

In general, it is not possible to have a regime of length scales over which nontrivial and different surface and mass fractal dimensions simultaneously pertain to the solid, although the general form for $S(Q)$ in this regime has been derived by Ball and Sinha ${ }^{30}$ and has the form

$$
S(Q) \sim 1 / Q^{\left(2 D-D_{s}\right)}
$$


In all known physically realizable cases, however, this result reduces to those discussed above. For instance, for a fractal aggregate of particles of dimension $D$, at length scales greater than the particle size $D_{3}=D$, the surface area contained inside a radius $R$ is obviously proportional to the number of particles inside this radius, and thus we obtain $S(Q)-Q^{-D}$. On the other hand for length scales smaller than the particle size, the particle looks solid and thus $D=3$, while $D_{s}$ is given by the particle surface fractal dimension and we recover $S(Q) \sim Q^{\left(-6-D_{2}\right)}$. A non-trivial limit occurs when $D, D_{3} \rightarrow 3$. This corresponds to the case of a porous solid which is sufficiently compacted that both the mass and the internal surface become uniformily spacefilling. Such solids should yield a scaling law of $S(Q)-Q^{-3}$ as also predicted by the theory of Wong and Bray ${ }^{24}$ but not the result of Bale and Schmidt. ${ }^{21}$

It is to be noted that the surface fractal dimension for the internal pore surface can in principle also be determined from the tiling definition of such a dimension, namely that the number of molecules of linear diameter $d$ adsorbed on such a surface is proportional to $\left(\mathrm{d}^{-\mathrm{D}_{s}}\right)$ assuming the adsorption is determined only by geometrical constraints. This idea has been used by Avnir and co-workers ${ }^{31}$ to derive $D_{1}$ for a variety of porous materials based on adsorption measurements. Unfortunately, this value of $D_{3}$ may be misleading (or even in some cases greater than 3, which is clearly non-physical) due to interaction effects between the adsorbate molecules and between these molecules and the surface. Scattering measurements of $D_{3}$ should be much more reliable since they are determined by purely geometrical factors.

\section{Small Angle Scattering Studies of Fluids Confined in Porous Media}

The behavior of fluids in confined pore spaces is of obvious interest and most conveniently susceptible to study by SANS techniques. This is because it is 
possible to fill the pores with a fluid whose SLD exactly matches that of the solid, thus removing the scattering from the pore structure itself, thereby reducing the 2phase system to a 1-phase system. The only fluctuations which will then scatter radiation are deviations from the average density, which will be fluctuations in the fluid itself.

A problem which has been studied by several groups by both SANS and light scattering techniques is that of the phase separation of a binary fluid mixture confined in a porous medium, such as Vycor glass or an aerogel. ${ }^{32+1}$ A convenient system for such studies is a mixture of water and $(2,6)$ lutidine, which in bulk has an inverted phase diagram (with a homogeneous phase at low temperatures, and phase separation occurring at higher temperatures). The critical concentration in the bulk mixture is $31.2 \%$ lutidine, and the critical temperature is $33^{\circ} \mathrm{C}$. In the vicinity of the critical point in the single phase region, a bulk mixture shows critical fluctuations obeying 3D Ising-like behavior. The behavior in Vycor glass as seen by light scattering ${ }^{37,41}$ is very different and indicative of the effects of confinement and preferential wetting of the pores (typically $80 \AA$ in diameter), with weak or nonexistent critical fluctuations and long-time relaxation and hysteresis effects with temperature in the 2-phase region. SANS data from such a system ${ }^{36,37}$ as a function of temperature is shown in Fig. 7. In order to study the concentration fluctuations in the fluid alone, without the complications from the scattering due to the solid/liquid contrast, the homogeneous phase was taken to be contrast-matched with the Vycor using the appropriate mixture of $\mathrm{H}_{2} \mathrm{O}, \mathrm{D}_{2} \mathrm{O}$ and lutidine. (The fact that Vycor preferentially absorbed lutidine from the supernatant solution complicated the task of ensuring that the final single-phase mixture inside the Vycor was nominally critical and contrast-matched, but this was achieved by a systematic study of different initial mixtures ${ }^{36}$ ). The "Vycor peak" at $Q \sim 0.025$ (see Fig. 2b) is absent due to the contrast- matching with the silica (see Fig. 7), but a peak at a larger value of $Q\left(-0.035 \mathrm{~A}^{-1}\right)$ was observed in the data. This peak was identified as due to 
a "skin" of lutidine-rich liquid adsorbed on the internal pore surfaces, as proved by a complementary experiment, in which a similar layer of hydrocarbon chains was attached to the internal pore surface of Vycor inside a contrast-matching solution of H/D toluene in the pores. The peak at the same $Q$ was clearly observed (see Fig. 8) and was used to subtract off the "skin" scattering from the observed $S(Q)$ from the Vycor/water/lutidine mixture i.e. from the observed data in Fig. 7. The remaining scattering was fitted by the sum of a Lorentzian to represent the critical fluctuations (which turned out to have a very small amplitude) and a Lorentzian-squared term (with a different length-scale) to represent the formation of microdomains of phaseseparated water-rich and lutidine-rich phases in the 2-phase region (see Eq.(34)). The fitted curves are shown in Fig. 7. A good fit was obtained, with the domain size saturating in the 2-phase region at the $80 \AA$ length-scale of the Vycor pores. This provided partial confirmation of the phase diagram obtained theoretically by Liu et al. $^{40}$ for a fluid mixture phase separating inside a finite tube. In their phase diagram (see Fig. 9), the system goes from a "tube" phase (lutidine-rich "skin" lining the pore walls) to a "capsule"-like phase ("skin" and capsules of water-rich phase within the tubes) as the temperature is raised into the 2-phase region.

A similar study was carried out by Frisken et al. ${ }^{39}$ in the much more open structures of a series of silica gels using a $\mathrm{D}_{2} \mathrm{O}$ lutidine mixture. In this case, the scattering was interpreted as that from a dispersed fractal structure of the silica framework, together with the associated static concentration variations induced in the fluid by the preference of the silica for wetting itself with lutidine, in addition to spontaneous critical fluctuations in the fluid. Thus this model is similar in spirit to the well-known "Random Field Ising Model" which was initially proposed for such systems. $^{32,33}$

Following Eq.(5) we may write

$$
S(Q)=\frac{1}{V}\left\langle\delta \rho(\mathbf{Q}) \delta \rho^{*}(\mathbf{Q})\right\rangle
$$


where $\delta \rho(\mathbf{Q})$ is the Fourier transform of the SLD fluctuation $\delta \rho(\mathbf{r})$. If $\phi_{s}(r), \phi_{1}(r), \phi_{w}(r)$ are respectively the local volume fractions of silica, lutidine and $D_{2} O$ and their fluctuations from the mean are $\delta \phi_{s}, \delta \phi_{1}, \delta \phi_{w}$, then

$$
\rho(\mathbf{Q})=\rho_{s} \phi_{s}(\mathbf{Q})+\rho_{1} \phi_{1}(\mathbf{Q})+\rho_{w} \phi_{w}(\mathbf{Q})
$$

where $\rho_{3} \rho_{1,} \rho_{w}$ are the SLD's of silica, lutidine and water respectively.

Since

$$
\delta \phi_{s}+\delta \phi_{1}+\delta \phi_{w}=0
$$

we obtain from Eq. (52)

$$
\delta \rho(\mathbf{Q})=\left(\rho_{s}-\rho_{w}\right) \delta \phi_{s}(\mathbf{Q})+\left(\rho_{1}-\rho_{w}\right) \delta \phi_{1}(\mathbf{Q})
$$

Let us ignore spontaneous critical concentration fluctuations in the liquid for the moment and concentrate on the response of the lutidine concentration to the silica concentration via the "wetting" interaction. Assuming linear response, this may formally be written as

$$
\delta \phi_{1}(\mathbf{Q})=\alpha(\mathbf{Q}, \mathbf{T}) \delta \phi_{s}(\mathbf{Q})
$$

where $\alpha(\bar{q}, T)$ may be written in the Ornstein-Zernike form

$$
\alpha(Q, T)=-\frac{\bar{\phi}_{1}}{\bar{\phi}_{1}+\bar{\phi}_{w}}+\frac{\alpha_{0}(T)}{1+Q^{2} \xi^{2}}
$$

(The constant term in Eq. (56) is there to account for the excluded volume decrease of lutidine concentration due to the presence of the silica, even in the absence of wetting, i.e., when $\alpha_{0}=0$ ).

Using Eqs. (54-56) in Eq. (51), we obtain

$$
S(Q)=\frac{1}{V}\left[\left(\rho_{s}-\rho_{w}\right)+\left(\rho_{1}-\rho_{w}\right) \alpha(Q, T)\right]^{2}\left(\delta \phi_{s}(Q) \delta \phi_{s}^{*}(Q)\right)
$$

But the scattering from the silica gel itself in pure $\mathrm{D}_{2} \mathrm{O}$ may be written as

$$
S_{s g}(Q)=\frac{1}{V}\left[\rho_{s}-\rho_{w}\right]^{2}\left\langle\delta \phi_{s}(Q) \delta \phi_{s}^{*}(Q)\right\rangle
$$


so that the scattering from the binary fluid mixture may be expressed in terms of $S_{s q}(Q)$ (measured in a separate experiment) and $\alpha(Q, T)$ as

$$
S(Q)=\left[1+\frac{\rho_{1}-\rho_{w}}{\rho_{s}-\rho_{w}} \alpha(Q, T)\right]^{2} S_{s g}(Q)
$$

To this must be added the pure critical fluctuations in the fluid given by

$$
S_{\text {crit }}(Q)=\left(\rho_{1}-\rho_{w}\right)^{2} \chi /\left(1+Q^{2} \xi^{2}\right)
$$

and a background term. Such an expression was found to provide an extremely good fit (Fig. 10) to the data for a wide range of temperatures and concentrations throughout the one-phase region of the pure system. The four fitting parameters used were $\chi, \xi, \alpha_{0}$ and a constant background term $B$.

The results showed that $\xi$ increased towards the critical temperature, as did $\alpha_{0}$ and $\chi$, although the accuracy was not sufficient to determine any critical exponents (in addition to the fact that the concentration of the "free" fluid, that which is not "frozen" in the wetting layers, is also changing with temperature). This behavior appears to be different to the behavior observed in Vycor. $\alpha_{0}$ was also found not to scale with $\chi$, which would be expected from a simple linear response theory, indicating that the wetting response of the fluid near the silica surface is probably non-linear as might be expected. In the 2-phase region, $S(\mathbf{Q})$ could be represented by

$$
S(Q)=\frac{C_{1}}{\left(1+Q^{2} \xi_{d}^{2}\right)^{2}}+C_{2} S_{s g}(Q)
$$

plus a constant background term, i.e., scattering due to the silica gel in an effective uniform fluid plus scattering from domains (see Eq. (34)) indicating that sharp interfaces between phase separated domains had formed. Eq. (61) is similar in spirit to the model of Debye et al., ${ }^{7}$ and the models used by Wong et al. ${ }^{42}$ and Lin et al. ${ }^{36}$ to describe frozen domains. $\xi_{d}$ was too large to measure accurately. Within the instrumental resolution, the first term in Eq. (61) effectively looked like Porod 
scattering. These domains also appeared to coarsen with increasing time, as observed by an increase in the amplitude of the $Q^{-1}$ term (proportional to the interfacial area by Eq. (31)).

The conformation of polymers confined inside pore spaces is also of interest. At first thought it would appear difficult to get a polymer whose equilibrium radius of gyration $R_{\mathbf{g}}$ in solution is greater than a typical pore size to enter the porous medium. However, it turns out, as predicted theoretically ${ }^{43}$ that from a sufficiently concentrated (semi-dilute or more concentrated) polymer solution osmotic pressure will force the polymer chains to enter the pores, the criterion being roughly that the correlation length $\xi$ in the semi-dilute solution be comparable to the pore size. This was observed in SANS experiments by $\mathrm{Lal}_{\text {et }}$ al. ${ }^{44}$ where polystyrene (PS) chains of equilibrium radius of gyration as large as $300 \AA$ were imbibed into cleaned Vycor glass from semi-dilute solutions in times ranging from hours (for the smallest chains) to 65 days (for the largest chains). This was verified by first studying the SANS from the virgin Vycor sample with a contrast-matched solvent (deuterated dichloromethane) in the pores (which showed no characteristic "Vycor peak") and comparing it with the imbibed Vycor in which the Vycor peak had reappeared, indicating that PS chains entering the Vycor had destroyed the contrast-matching condition.

For the SANS experiments a mixture of hydrogenated and deuterated PS chains of equal molecular weights (MW) (h-PS and d-PS respectively), and hydrogenated and deuterated toluene solvent (h-toluene and d-toluene) was used. The SANS experiment can be made sensitive to the conformation of individual PS chains inside the Vycor if the following two conditions are simultaneously met: 1) The h-, d-toluene mixture exactly contrast matches the silica of the Vycor. (This was determined by careful subsidiary contrast matching experiments using varying $\mathrm{h}-$, and d-toluene concentrations.) 
2) The concentration of $h-P S$ and d-PS chains is chosen such that the averaged SLD of the chains exactly matches that of the solvent.

Under these conditions it may be shown ${ }^{44}$ that only single chain fluctuations will contribute to the observed $S(Q)$ which thus measures the form-factor or conformation of simple chains. Experiments were done for a variety of PS molecular weights. A typical $\mathrm{S}(\mathrm{Q})$ curve for $\mathrm{PS}$ of $\mathrm{MW} 75 \mathrm{~K}$ in toluene in Vycor is shown in Fig. 11. The results were compared with the $S(Q)$ for the same chains in dilute solution in toluene (i.e. "free" chains). A Debye-function fit (appropriate to faussian chains in a good solvent) ${ }^{43}$ was made to both sets of data. Such a procedure is valid for obtaining a radius of gyration from the small $Q$ region where the fit is reasonably good, although at large $Q$ both curves deviate. The radius of gyration of the confined chains were obtained to be always smaller than the radius of gyration of the free chains in the equivalent bulk solution. The free chains in dilute solution yield an $S(Q)$ that deviates from the $1 / Q^{2}$ Debye-like behavior due to excluded volume effects (yielding $Q^{-1 / v}$, where $V$ is the Flory exponent ${ }^{43}$ ). The chains in the Vycor deviate due to conformational changes arising from confinement in the tube-like pores. These were interpreted in terms of the theory developed by Daoud and de Gennes ${ }^{45}$ and Brochard and de Gennes. ${ }^{46}$ Briefly, the theory takes into account four main effects which govern the behavior of chains confined in nonadsorbing cylindrical pores. These are the confinement of the chains by the cylinder walls, which squeezes the chains laterally, the intrachain excluded volume effect which swells the chains and stretches them along the cylinder axis, the entropic elasticity of the chains which limits this stretching, and the interchain interactions which may also lead to segregation of the chains. Thus the polymer chains are stretched out into "cigars" along the tubes, which may at high concentrations segregate from each other (strong confinement limit) or overlap in an entangled manner (moderate confinement regime) and thus no longer be stretched along the cylinder axis. In the latter cases $R_{g z}^{2}=R_{g}^{2} / 3$, where $R_{g z}$ is the radius of gyration 
along the cylinder axis, and $\mathrm{R}_{\mathbf{g}}$ is that of the chain in bulk solution. One then has the relation

$$
R_{g v}^{2}=D^{2} / 8+R_{g z}^{2}
$$

where $R_{x y}$ is the radius of gyration as measured in the Vycor pore space and D is the pore diameter of Vycor (known to be $\sim 70 \AA$ ). Thus Eq.(62) provides a method for testing the relationship of the measured radii of gyration of the individual chains in Vycor and in the bulk solution, and was found to be satisfied extremely well for the different molecular weights studied. Thus one can conclude that the chains were in the conformation of ideal overlapping squeezed cigars. This conclusion was confirmed by also fitting the $S(Q)$ of the individual chains in the regime $D<Q^{-1}<R_{\text {e }}$ to a "cylindrical" Guinier model.

\section{Surfaces and Interfaces}

To study inhomogeneities (such as roughness, islands on clusters, capillary wave fluctuations, etc.) on surfaces and interfaces, the technique analogous to small-angle scattering (SAS) is off-specular $\mathrm{X}$-ray or neutron reflection at small angles. (Specular reflectivity studies, which in general measure the laterally averaged density profile normal to the surface are discussed in the lecture by McMorrow). As we shall see, off-specular or diffuse surface scattering probes the height-height correlation function for surface fluctuations, just as as SAS probes density-density correlation functions in the bulk. ${ }^{47}$

To see this, consider a single interface between two media of SLD's $\rho_{1} \rho_{2}$ respectively so that the contrast $\Delta \rho=\left(\rho_{1}-\rho_{2}\right)$. Since $Q$ is small, we may again neglect the atomic or molecular structure of the media and assume that the only density fluctuation in the system is across the interface. Then, from the Born Approximation expression for $S(Q)$ given by Eq. (5), we may obtain by integrating over the $\mathrm{z}$ coordinates, 


$$
S(Q)=\frac{(\Delta \rho)^{2}}{Q_{z}^{2}} \iiint \int d x d y d x^{\prime} d y^{\prime} e^{i Q_{z}\left(\delta z(x, y)-\delta z\left(x^{\prime}, y^{\prime}\right)\right)} e^{i\left[Q_{x}\left(x-x^{\prime}\right)+Q_{y}\left(y-y^{\prime}\right]\right.}
$$

where $\hat{z}$ represents the axis normal to the average surface and $\delta z(x, y)$ is the height fluctuation at lateral position $(x, y)$ about this average surface. Let us assume such fluctuations have a random Gaussian distribution. Carrying out a statistical average yields an integrand which depends only on the relative separation $(X, Y)$ of the coordinates $(x, y)$ and $\left(x^{\prime}, y^{\prime}\right)$ and we obtain

$$
S(\mathbf{Q})=A \frac{(\Delta \rho)^{2}}{Q_{z}{ }^{2}} \iint d X d Y e^{-\frac{1}{2} Q_{z}^{2} g(R)} e^{-i\left(Q_{x} X+Q_{y} Y\right)}
$$

where

$$
g(R)=\left\langle[\delta z(X, Y)-\delta z(0,0)]^{2}\right\rangle
$$

$R=(X, Y)$ and $A$ is the surface area. Now $g(R)$ can be written as

$$
g(\mathbf{R})=2 \sigma^{2}-2 C(\mathbf{R})
$$

where $\sigma^{2}$ is the mean-square roughness of the surface $\left\langle[\delta z(x, y)]^{2}\right\rangle$, and $C(R)$ is the height-height correlation function

$$
C(R)=\langle\delta z(X, Y) \delta z(0,0)\rangle
$$

By Eqs. (65) and (66), we obtain

$$
S(Q)=A \frac{(\Delta \rho)}{Q_{z}^{2}} e^{-Q_{z}^{2} \sigma^{2}} \iint d X d Y e^{Q_{z}^{2} C(R)} e^{-i\left(Q_{x} X+Q_{y} y\right)}
$$

Since $C(\mathbf{R}) \rightarrow \infty$, the integral in Eq. (68) contains a delta function corresponding to specular reflectivity, which can be explicitly removed by subtracting unity from the integrand in Eq. (68) to yield the diffuse component.

Then, 


$$
S_{\text {diff }}(Q)=A \frac{(\Delta \rho)^{2}}{Q_{z}^{2}} e^{-Q_{z}^{2} \sigma^{2}} \iint d X d Y\left[e^{Q_{z}^{2} C(R)}-1\right] e^{-i\left(Q_{x} X+Q_{y} Y\right)}
$$

Quite often, $Q_{y}$ (the component of $\mathbf{Q}$ out of the scattering plane) is effectively integrated over by having wide open slits in this direction, in which case Eq. (69) involves only a ID integral over $X$. The generalization of Eq. (69) to multiple interfaces (for thin films and multilayers) is straightforward ${ }^{43-53}$ but will not be discussed here. For scattering at very small $Q_{2}$, the Born Approximation fails and must be replaced by the Distorted Wave Born Approximation (DWBA), ${ }^{47}$ which leads to a modification of Eq. (69). The reader is referred to refs. [47], [48] and [58] for futher details.

The assumption of a self-affine fractal surface as given by Eq. (47) (modified for a finite roughness correlation length $\xi$ yields

$$
g(R)=2 \sigma^{2}\left(1-e^{-(R / \xi)^{2 h}}\right)
$$

which leads to an explicit form for $C(R)$

$$
C(R)=\sigma^{2} e^{-(R / \xi)^{2 h}}
$$

involving basically 3 parameters to characterize the surface roughness, namely $\sigma, \xi$ and $h$. This often works reasonably well in describing the results of surface roughness measurements by either scattering or AFM or STM methods. ${ }^{54.55}$ For liquid surfaces on the other hand a logarithmic form of $C(R)$ usually holds.

\section{Scattering by Non-Gaussian Surface Fluctuations}

Many kinds of surfaces have specific surface features that cannot be discussed within the random gaussian self-affine model of roughness discussed in Section 4 . Examples are surfaces with islands of fixed or variable heights above the reference surface, surfaces with pits (as in the case of corrosion), surfaces with steps, etc. In order to discuss the scattering from such surfaces in the Born Approximation, we must go back to the basic formula for $S(Q)$ given in Eq. (63). Let us for the 
moment imagine that the height function $\delta z(x, y)$ for the interface has a bimodal distribution, being 0 with probability $P_{1}(x, y)$ and $\Delta$ with probability $P_{2}(x, y)=1$ $P_{1}(x, y)$. (This corresponds to islands of fixed height $\Delta$ across the interface). Then Eq. (63) may be written as

$$
\begin{aligned}
& S(q)=\frac{A(\Delta p)^{2}}{q_{z}^{2}} \iint d x d y\left\{\left[P_{1}(0,0) P_{1}(x, y)+P_{2}(0,0) P_{2}(x, y)\right]+P_{1}(0,0) P_{2}(x, y) e^{-i q_{2} \Delta}+P_{2}(0,0) P_{1}(x, y) e^{i q_{2} \Delta}\right\} \\
& X e^{-i\left(Q_{2} X+Q_{z} Y\right.}
\end{aligned}
$$

Writing $\phi$ for the fractional coverage of islands, we may express the above in terms of the two-dimensional analogue $\gamma_{0}(x, y)$ of the Debye correlation function related to the probability of crossing over from no island to an island within a relative separation of $(x, y)$, and obtain

$$
S(Q)=S_{\text {spec }}(Q)+S_{\text {dif }}(Q)
$$

where

$$
S_{\text {spec }}(Q)=\frac{A 4 \pi^{2}(\Delta \rho)^{2}}{Q_{z}{ }^{2}} \delta(Q x) \delta(Q y)\left[1-4 \phi(1-\phi) \operatorname{Sin}^{2}\left(Q_{z}{ }^{2} \Delta / 2\right]\right.
$$

and

$$
S_{\text {diff }}(Q)=\frac{A(\Delta \rho)^{2}}{Q_{z}^{2}}(1-\phi) \operatorname{Sin}^{2}\left(Q_{z}{ }^{2} \Delta / 2\right) \iint d x d y \gamma_{0}(x, y) e^{-i\left(Q_{x} x+Q_{y} Y\right)}
$$

Note that the longitudinal diffuse scattering $\left(Q_{x}, Q_{y} \cong 0\right)$ has a modulation along $Q_{z}$ with period $(2 \pi / \Delta)$ which is exactly out of phase with a similar modulation in the specular reflectivity. This is in contrast to the case of conformal roughness, where the Kiessig fringes in the specular and the diffuse are in phase. The above theory can be easily generalized to the case of a film with islands deposited on a substrate, and to include roughness fluctuations as well. The expression for the specular reflectivity may be written as 


$$
\begin{aligned}
& R\left(Q_{z}\right)=\frac{16 \pi^{2}}{Q_{z}{ }^{4}}\left\{\rho_{i}{ }^{2} e^{-Q_{z}{ }^{2} \sigma_{1}{ }^{2}}\left[1-4 \phi(1-\phi) \operatorname{Sin}^{2}\left(Q_{z} \Delta / 2\right)\right]+\left(\rho_{2}-\rho_{1}\right)^{2} e^{-Q_{z}{ }^{2} \sigma_{2}{ }^{2}}\right. \\
& +2 \rho_{1}\left(\rho_{1}-\rho_{2}\right) e^{\frac{1}{2} Q_{z}{ }^{2}\left(\sigma_{1}{ }^{2}+\sigma_{2}{ }^{2}\right)}\left[\phi \operatorname{Cos}\left(Q_{z}(t+\Delta)+(1-\phi) \operatorname{Cos}\left(Q_{z} t\right)\right]\right\}
\end{aligned}
$$

where $t$ is the total film thickness, $\rho_{1}$ is the film electron density, $\rho_{2}$ that of the substrate and $\sigma_{1}, \sigma_{2}$ are respectively the roughness values at the film/air and film/substrate interfaces. This reflectivity expression yields both the rapid Kiessig fringes, as well as modulations due to the islands on the surface. Fig. 12 shows the specular reflectivity and longitudinal diffuse scattering from a polymer film decorated with such islands, where both the Kiessig fringes (which appear in phase in the specular and the diffuse scattering due to conformal roughness of the film and substrate) and the "island modulations " (which are out of phase in the specular and in the diffuse) are observed. ${ }^{\text {s6 }} \mathrm{Fig} .13$ shows transverse diffuse scans (rocking curves) obtained for another system studied, namely a copper film in contact with an electrolyte in an electrochemical cell to which a negative (oxidizing) voltage is applied, as a function of the time for the applied voltage. ${ }^{57}$ It may be seen that side peaks grow in the diffuse scattering on either side of the specular reflection. These are a consequence of the pit correlations, which are reflected in a peak in the Fourier transform of the $\gamma_{0}(x, y)$ function. 


\section{References}

1. A. Guinier and D.C. Fournet, "Small-angle Scattering of X-rays," (Wiley, New York, 1955).

2. O. Glatter and O. Kratky, "Small-angle X-ray Scattering" (Academic Press, New York, 1982);

3. T.P. Russell "Small-angle Scattering at Synchrotron Radiation Sources," in Handbook of Synchrotron Radiation, Vol. 3, (G. Brown and D.E. Moncton, Eds.) North Holland, Amsterdam (1991).

4. L.A. Feigin and D.I. Svergun, "Structure Analysis by Small Angle X-ray and Neutron Scattering," (Plenum Press, New York, 1987).

5. G.E. Bacon, "Neutron Diffraction" (3rd edition) Clarendon Press, Oxford, 1975.

6. G.L.Squires, "Introduction to the Theory of Thermal Neutron Scattering," Cambridge University Press, 1978.

7. P. Debye, H.R. Anderson and H. Brumberger, J.Appl. Phys. 28. 679 (1957).

8. J.M. Drake, P. Levitz and S. Sinha, Mat. Res. Soc. Symp. Proc. 73, 305 (1986).

9. D.W.Schaefer, B.C. Bunker and J.P. Wilcoxon, Phys.Rev.Lett. 58, 284 (1987).

10. P. Wiltzius, F.S. Bates, S.B. Dierker and G. Wignall, Phys.Rev.A 36, 2991 (1987).

11. P. Levitz, G. Ehret, S.K. Sinha and J.M. Drake, J.Chem. Phys. 25, 6151 (1991).

12. J. Mering and D. Tchoubar, J.Appl.Cryst. 1, 153 (1968); D. Tchoubar and J. Mering, J.Appl.Cryst. 2, 128 (1969).

13. M.Y. Lin and S.K. Sinha, Mat.Res.Soc.Symp.Proc. 195, 485 (1990). 
14. N. Berk, Phys.Rev.Lett. 58, 2718 (1987); Phys.Rev.A 44, 5069 (1991).

15. M. Teubner and R. Strey, J.Chem.Phys. 87,3195 (1987).

16. S.H. Chen, D.D. Lee, K. Kimishima, H. Jinnai and T. Hashimoto, Phys.Rev.E 54, 6526 (1996).

17. J-C. Li and D.K. Ross, J.Phys.Condens.Matter 6, 352 (1994).

18. B.B. Mandelbrodt, The Fractal Geometry of Nature (Freeman, New York, 1982).

19. S.K. Sinha, T. Freltoft and J. Kjems, in Kinetics of Agaregation and Gelation (Ed. F. Family and D. Landau) North-Holland, Amsterdam, 1984; pp 87-90.

20. T. Freltof, J.K. Kjems and S.K. Sinha, Phys. Rev. B 33, 269 (1986).

21. H.D. Bale and P.W. Schmidt, Phys.Rev.Lett. 53,596 (1984).

22. B.J. Frisken, F.Ferri and D.S. Cannell, Phys. Rev. Lett. 66, 2754 (1991).

23. G. Vacca, W.B. Dozier, A. Ellison, J.M.Drake and S.K. Sinha (unpublished).

24. P.-z. Wong and A. Bray, J.Appl.Cryst. 21,786 (1988); Phys.Rev.B 37. 7751 (1988).

25. D.W. Schaefer, J.E. Martin, P. Wiltzius and D.S. Cannell, Phys.Rev.Lett. 52, 2371 (1984); A.J. Hurd, D.W. Schaefer, D.M. Smith, S.B. Ross, A. Lemauté, and S. Spooner, Phys.Rev.B 39 9742 (1989).

26. P.W.Schmidt, H. Kaiser, A. Hohr, J.S. Lin, H.B. Neumann and D. Avnir, J. Chem. Phys. 20, 5016 (1989).

27. cf: P.-z. Wong, Phys.Today 41 (12),24 (1988) and references therein.

28. S.K. Sinha, E.B. Sirota, S. Garoff and H.B. Stanley, Phys.Rev. B 38, 2297 (1988).

29. P.-z. Wong, Phys. Rev. B 32, 7417 (1985).

30. R.C. Ball and S.K. Sinha, (unpublished); S.K. Sinha, Physica D 38, 310 (1989). 
31. D. Avnir, D. Farin, P. Peiffer, J.Chem.Phys. 79, 3566 (1983); Nature (London), 308, 261 (1984).

32. F. Brochard and P.G.de Gennes, J.Phys.Lett. 44, L-785 (1983).

33. D. Andelman and J.F. Joanny in Scaling Phenomena in Disordered Systems (ed. R. Rynn and A. Skjeltarp) Plenum, New York 1985, p.163.

34. W.I. Goldberg in Scaling Phenomena in Disondered Systems, (ed. R. Pynn and A. Skjeltorp) Plenum, New York 1985, p.151.

35. M.C. Goh, W.J. Goldburg \& C.M. Knobler, Phys.Rev.Lett. 58, 1008 (1987).

36. M.Y. Lin, S.K. Sinha, J.M. Drake, X.L. Wu, P. Thiyagarajan and H.B. Stanley, Phys.Rev.Lett. 72,2207 (1994).

37. S.B. Dierker and P. Wiltzius, Phys.Rev.Lett. 58, 1865 (1987); ibid 62,804 (1989); ibid 66, 1185 (1991).

38. B.J. Frisken and D.S. Cannell, Phys.Rev.Lett. 62,632 (1992).

39. B.J. Frisken, D.S. Cannell, M.Y. Lin and S.K. Sinha, Phys.Rev.E 51 , 5866 (1995).

40. A.J. Liu and G. Grest, Phys.Rev.A 44, R7894 (1991); L. Monette, A.J. Liu, and G. Grest, Phys.Rev.A 46, 7614 (1992); A.J. Liu, et al., Phys.Rev.Lett. 65, 1897 (1990).

41. J.V. Maher, W.I. Goldberg, D.W. Pohl and M. Lenz, Phys.Rev.Lett. 53, 60 (1984).

42. P.-z. Wong, J.W. Cable, and P. Dimon, J. Appl. Phys. 55, 2377 (1984).

43. P.G. de Gennes, Scaling Concepts in Polymer Physics (Cornell University Press, Ithaca, NY, 1985) 95.

44. J. Lal, S.K. Sinha and L. Auvray, J. de Physique II,Z, 1597 (1997).

45. M. Daoud and P.G. de Gennes, J. de Physique 38, 85 (1977).

46. F. Brochard and P.G. de Gennes, J. de Physique 40, L-399 (1979). 
47. S.K. Sinha, E.B. Sirota, S. Garoff and H.B. Stanley, Phys. Rev. B 38:2297 (1988); P.Z. Wong and A. Bray, Phys. Rev. B 37:7751 (1989).

48. R. Pynn, Phys. Rev. B 45:602 (1992); R. Pynn and S. Baker, Physica B 198:1 (1994).

49. J. Daillant and O. Bélorgey, J. Chem. Phys. $97: 5824$ (1992); ibid. 97:5837 (1992).

50. D.E. Savage, J. Leiner, N. Schimke, Y.H. Phang, T. Jankowski, J. Jacobs, R. Kariotis and M.G. Lagally, J. Appl. Phys. 69:1411 (1991); D.E. Savage, N. Schimke, Y.-N. Phang and M.G. Lagally, J. Appl. Phys. 71:3282 (1992).

51. M.K. Sanyal, S.K. Sinha, A. Gibaud, S.K. Satija, C.F. Majkrzak, and H. Homma, Surface X-ray and Neutron Scattering, H. Zabel and I.K. Robinson, Eds. (Springer-Verlag, Berlin, Heidelberg, 91 (1992); M.K. Sanyal et al in K. Liang, M.P. Anderson, R.F. Bruinsma and G. Scoles, Eds.,Mat.Res.Symp.Proc. 237:393 (1992).

52. S.K. Sinha, J. Phys. III, France 4:1543 (1994).

S.K. Sinha et al, Physica B 198:72 (1994).

W. Press et al. Physics B 198:42 (1994); M. Wormington, Philos. Mag. Lett. 74:211 (1996); see also Ref. [11].

55. T. Salditt, T.H. Metzger and J. Peisl Phys. Rev. Lett. 73:2228 (1994).

56. S.K. Sinha, Y.P. Feng, C.A. Melendres, D.D. Lee, T.P. Russell, S.K. Satija, E.B. Sirota and M.K. Sanyal, Physica A $231: 99$ (1996).

57. C.A. Melendres, Y.P. Feng, D.D. Lee and S.K. Sinha, J. Electrochem. Soc. 142:L119, (1995); Y.P. Feng, S.K. Sinha, C.A. Melendres and D.D. Lee, Physica B 221:251 (1996).

58. V. Holy and T. Baumbach, Phys. Rev. B 49:10688 (1994). 


\section{Figure Captions}

Fig.1

Schematic of chord distribution model representing a bicontinuous medium composed of media 1,2 .

Fig. 2

(a) Scaled $S(Q)$ for two silicagels on a log-log plot, showing asymptotic $Q^{-1}$ behavior (from Ref. [12]). Unfilled squares represent unscaled data for silicagel of nominally $40 \AA$ pore size; asterisks denote rescaled data for silicagel of nominally 4000 A pore size. (b) $S(Q)$ for dry Vycor glass on a log-log plot (open circles) showing a deviation from Porod's Law above $0.05 \AA^{-1}$ with $Q^{-3.7}$ behavior (from Ref. [17]).

Fig.3 Electron Micrograph of Silicagel Si-4000 at (from Ref. [12]).

Fig4 Transmission electron micrograph (TEM) of a thin section of Vycor glass. (From Ref. [15]).

Fig.5 $\mathrm{S}(\mathrm{Q})$ for a series of silica gels made in $\mathrm{D}_{2} \mathrm{O}$ at different $\mathrm{pH}$, as indicated. The silica volume fraction for esch sample is also denoted on the graph. The solid curves are fits of Eq. (49) to the data with $\mathrm{D}=2.22,2.13$ and 2.03 for the $\mathrm{pH} 5.35,5.45$ and 5.99 samples respectively (From Ref. [26]).

Fig.6 Schematic diagram for $S(Q)$ of a system of fractal clusters. $\xi$ is the cluster size and a is the size of a constituent solid particle in the cluster.

Fig.7 $S(Q)$ (arbitrary units) for a contrast-matching binary lutidine/water mixture near the critical concentration inside Vycor glass, as a function of temperature. (From Ref. [44]). 


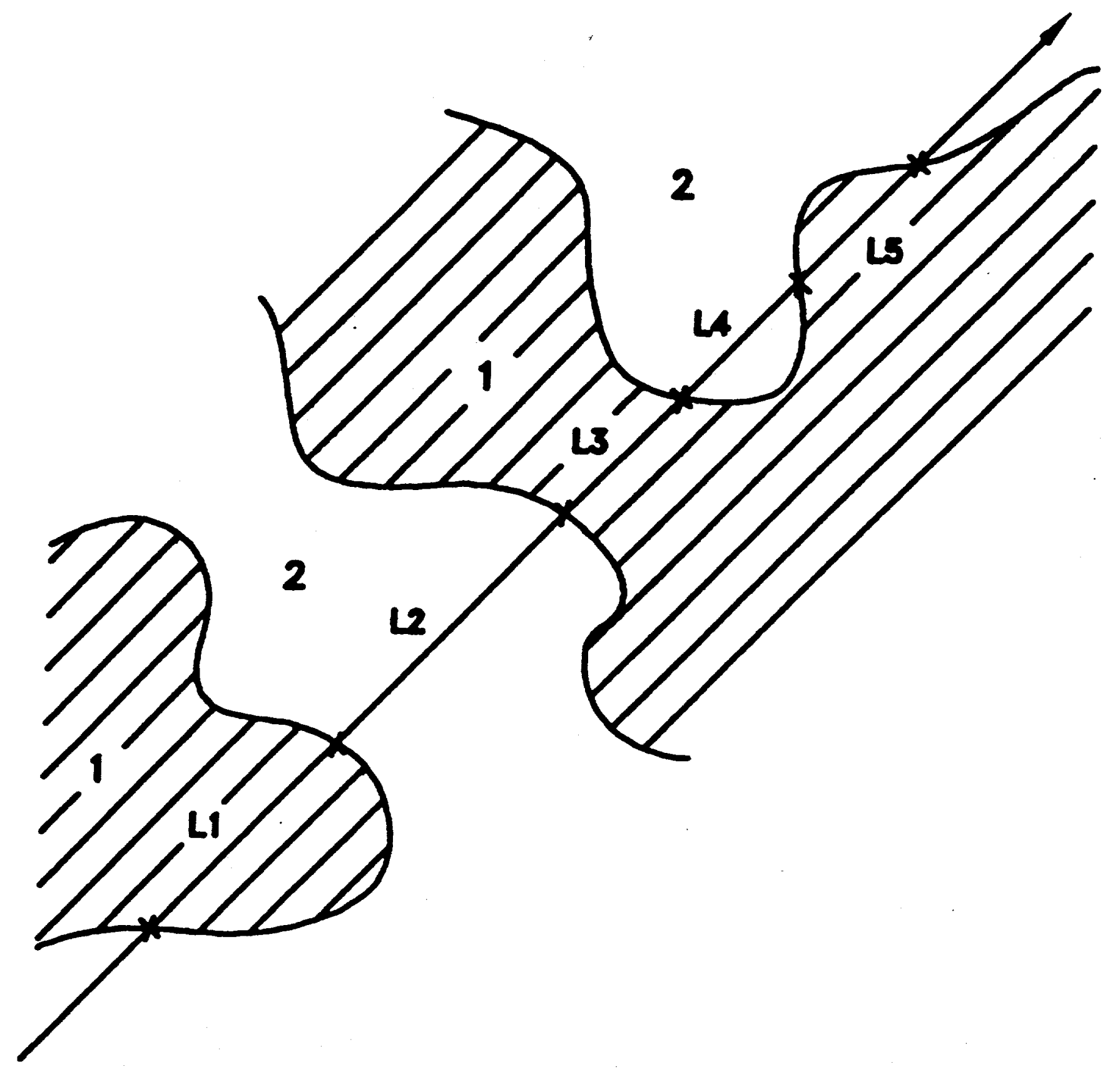




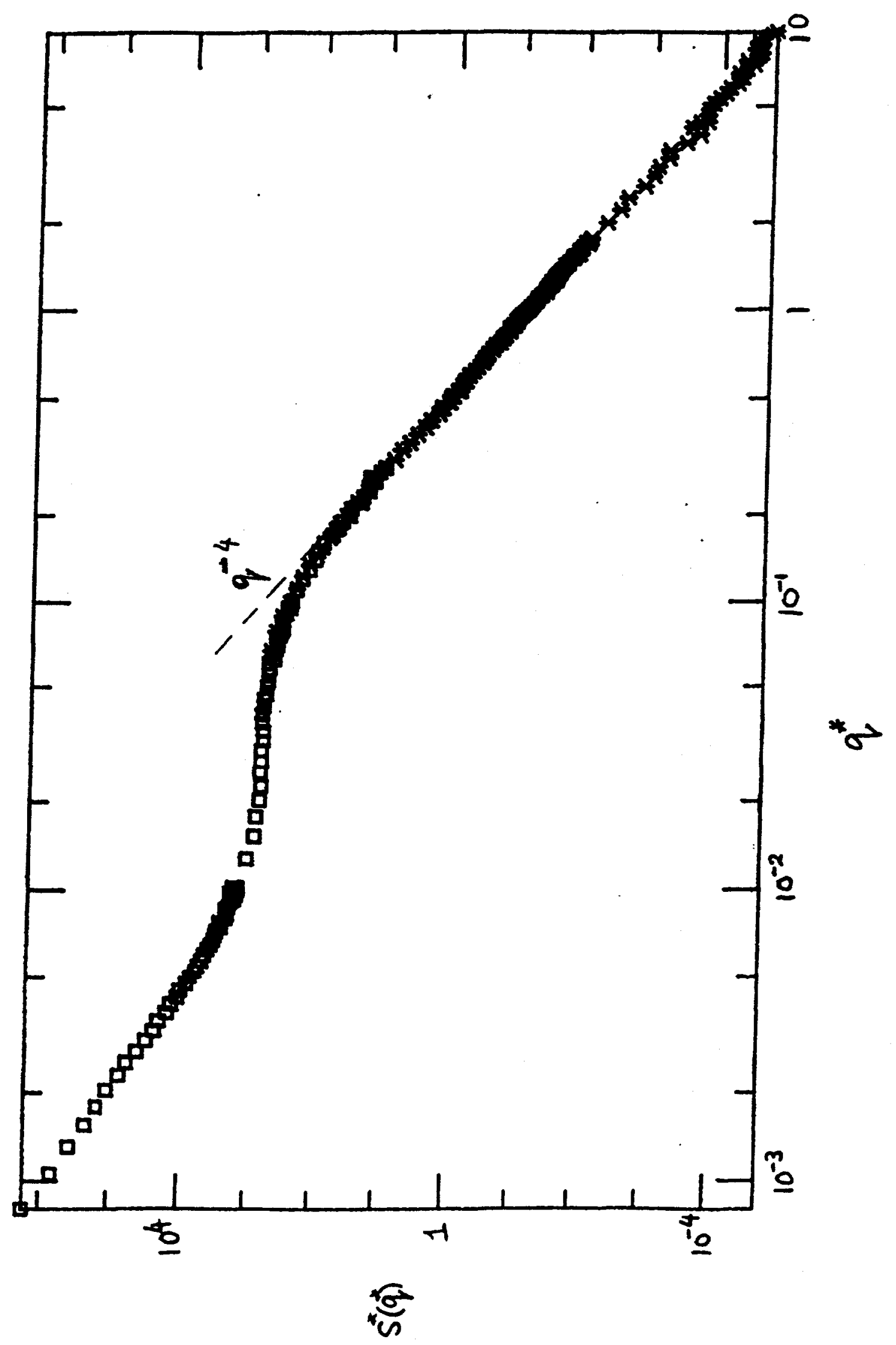




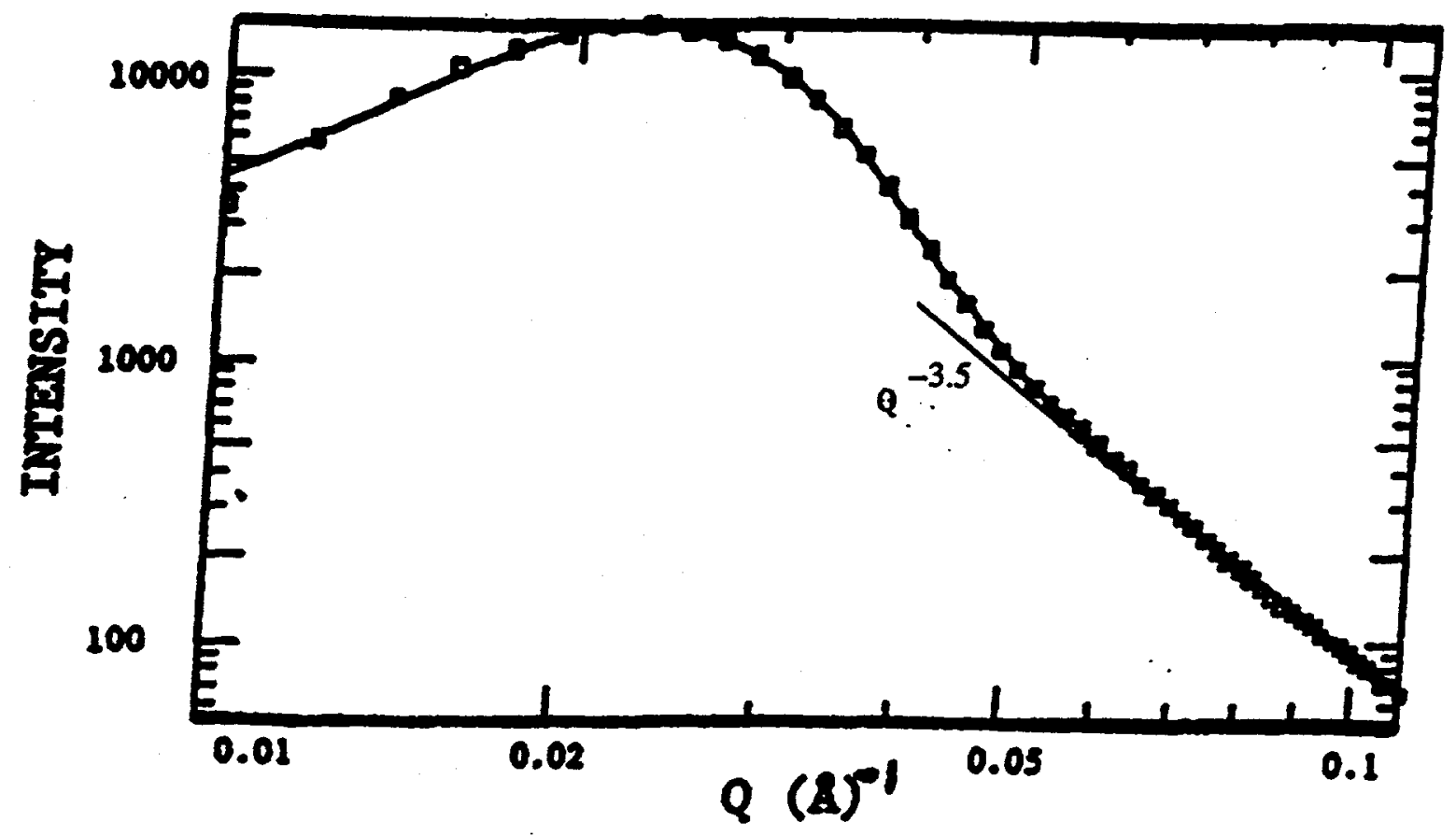

Fig 20 


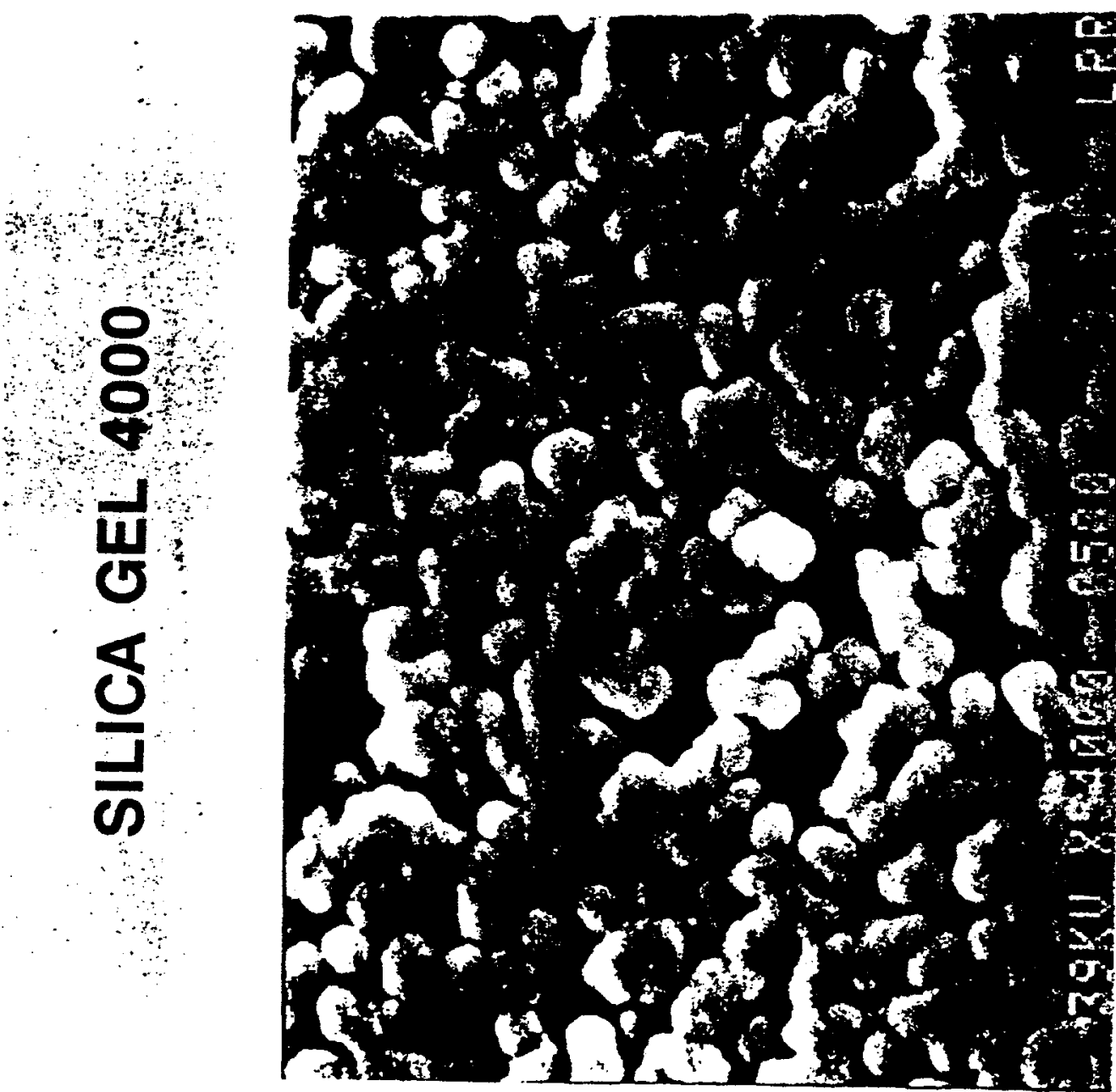



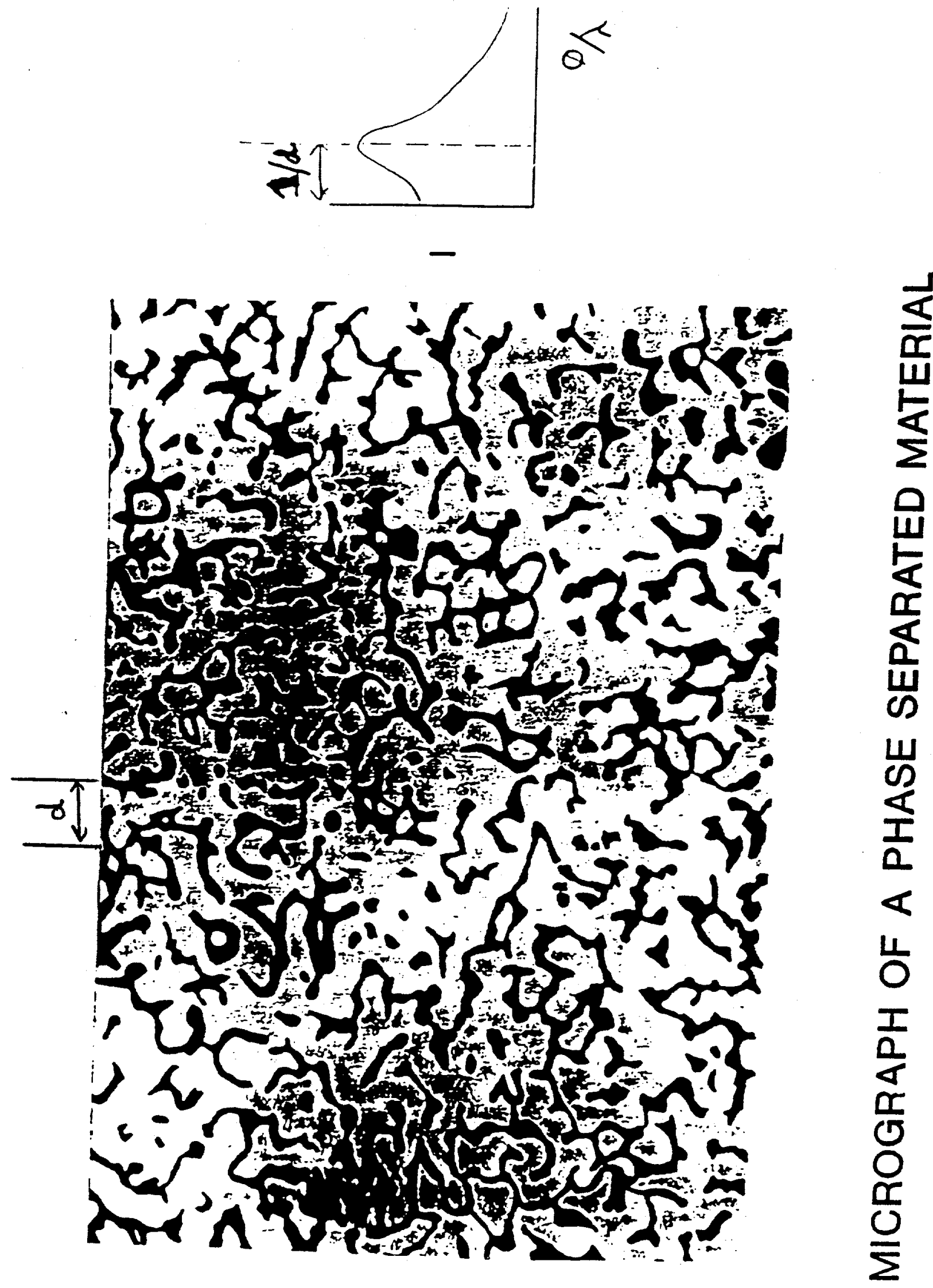


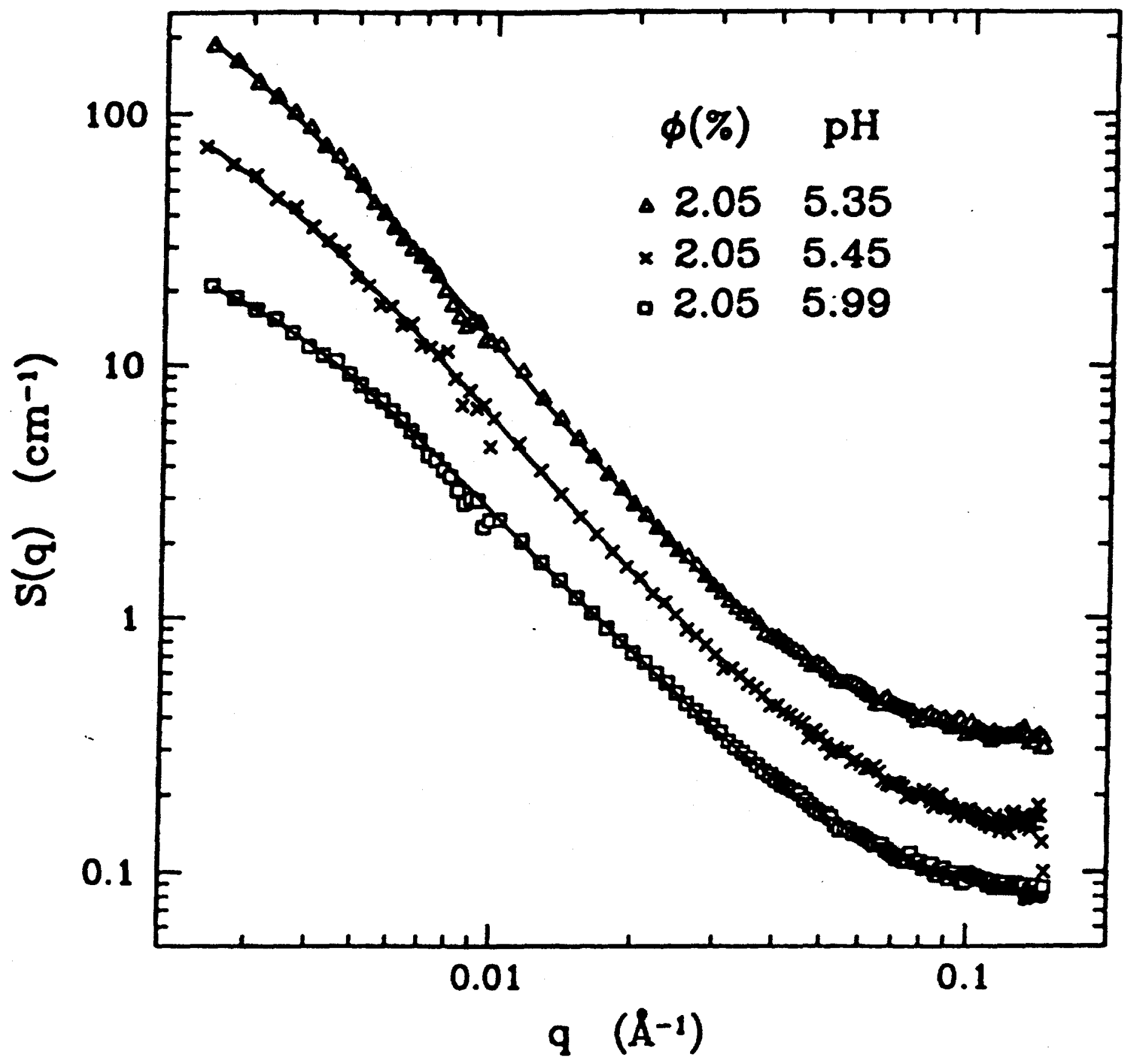




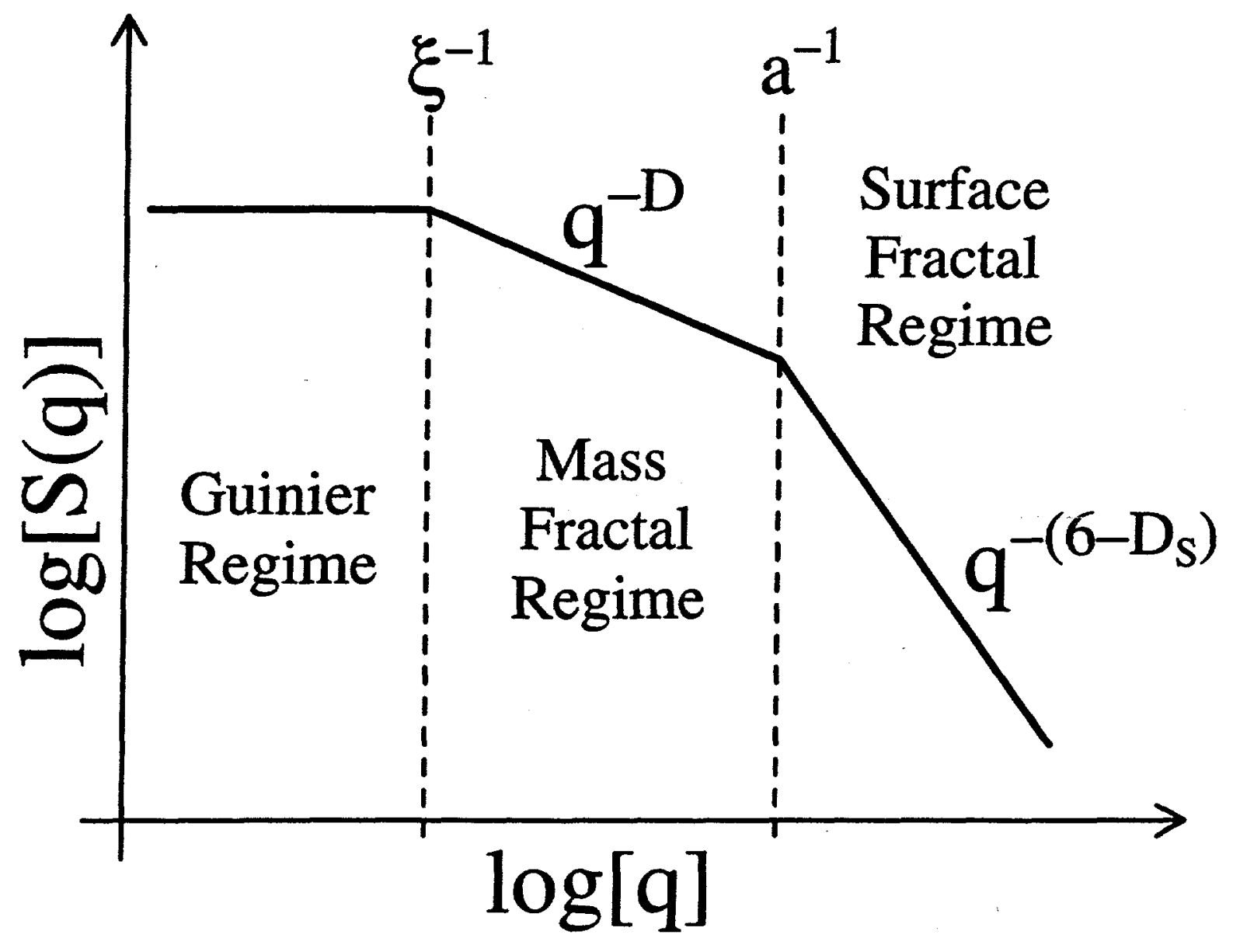

$7+16$ 


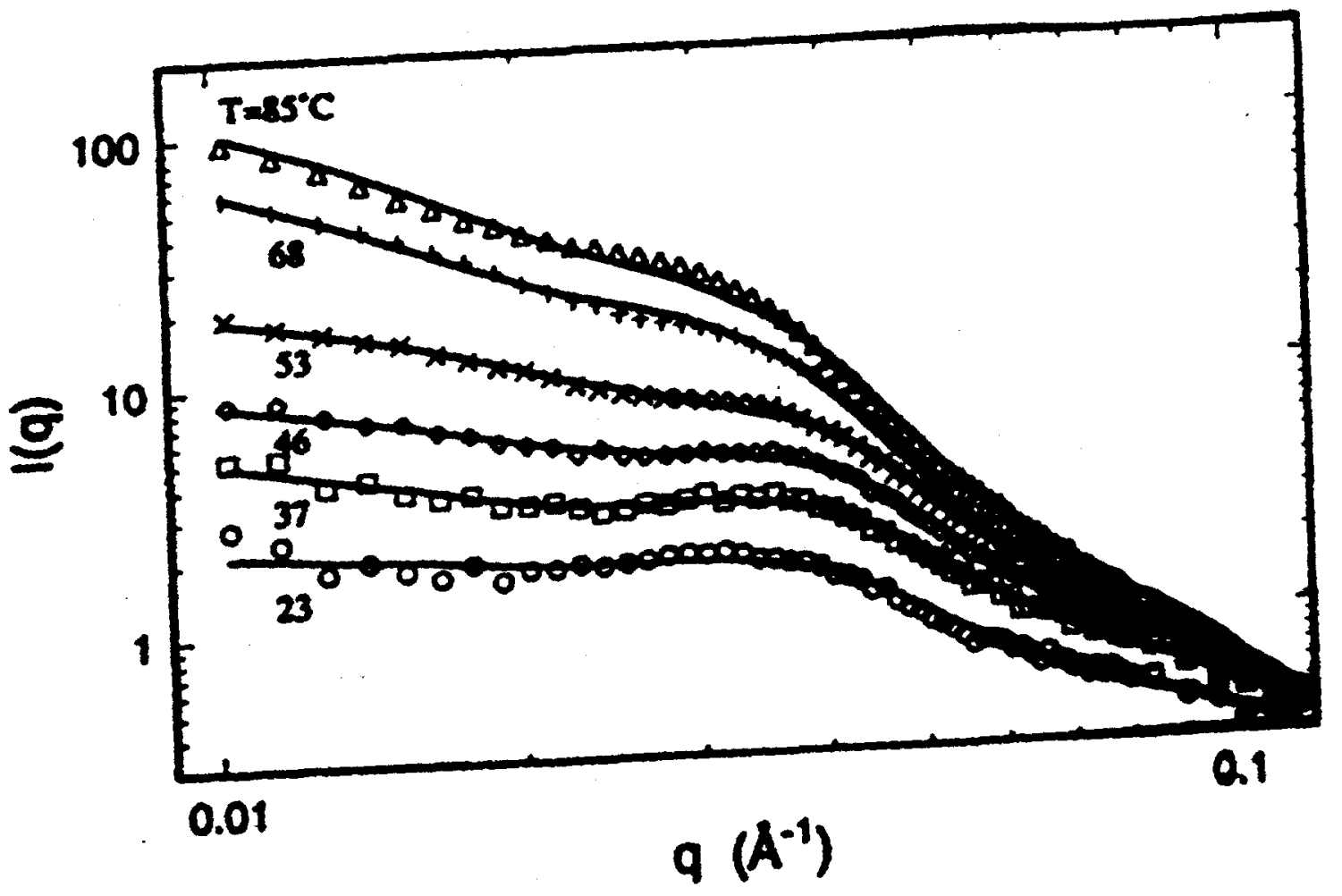

$7: 97$ 


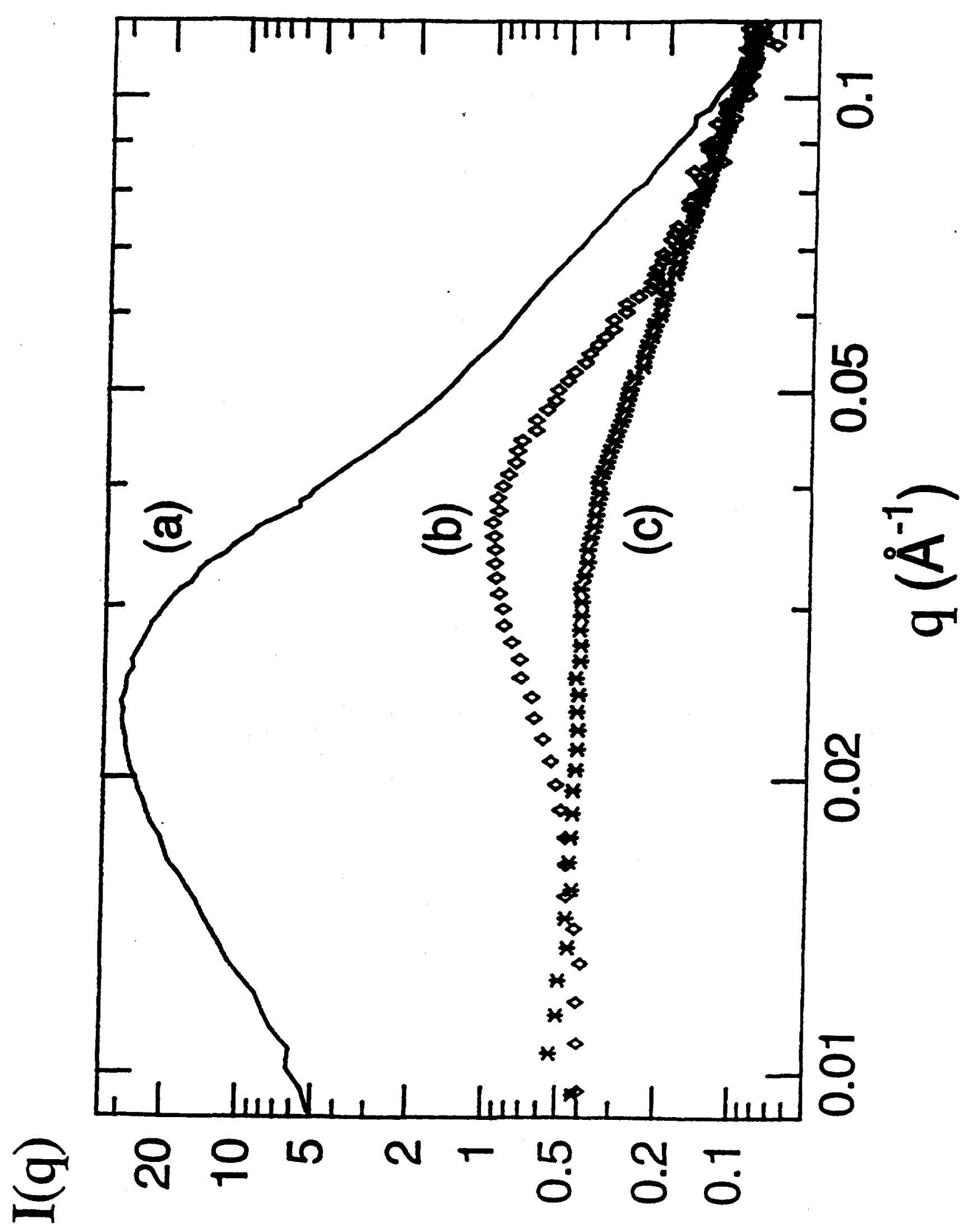




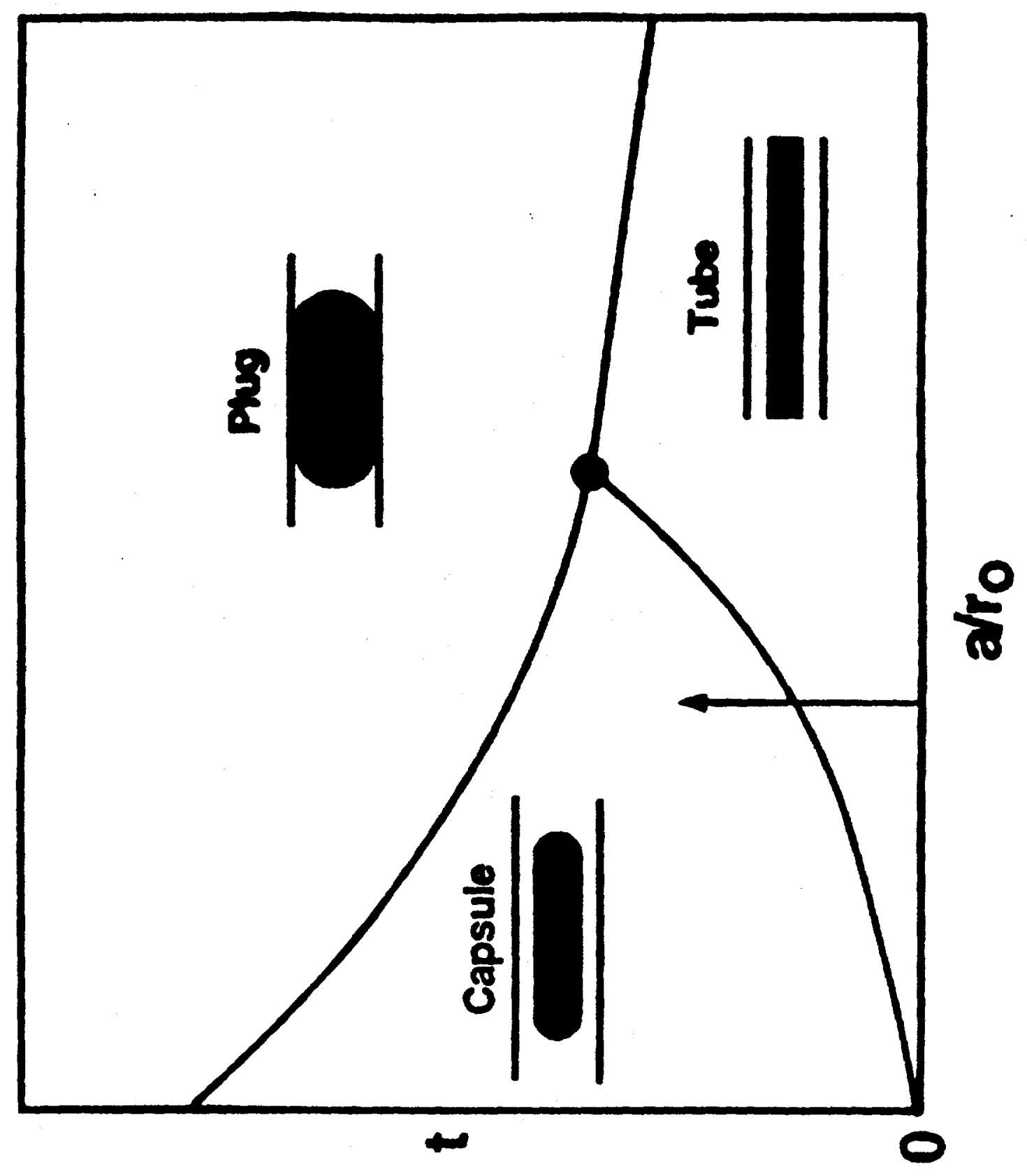




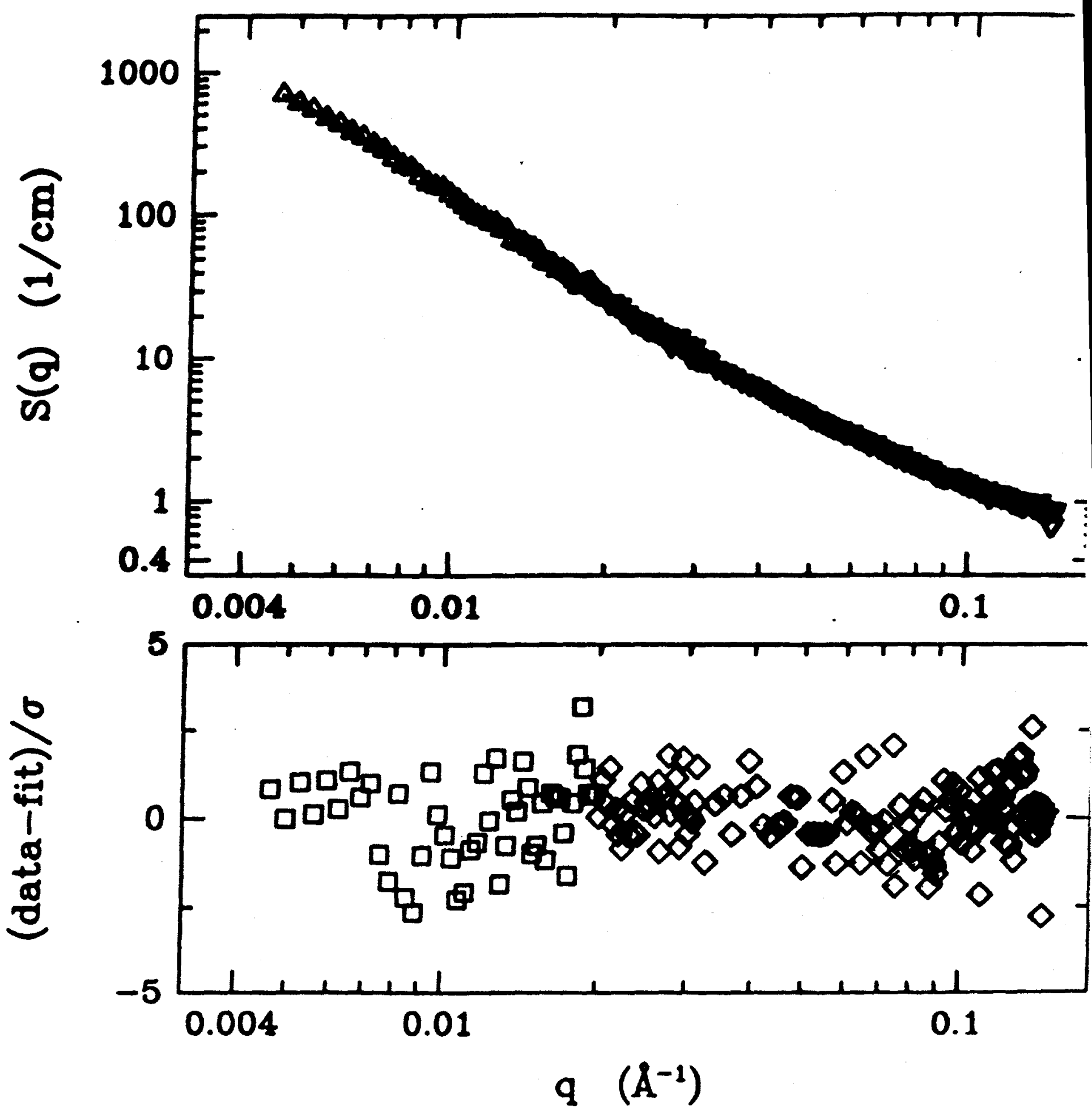




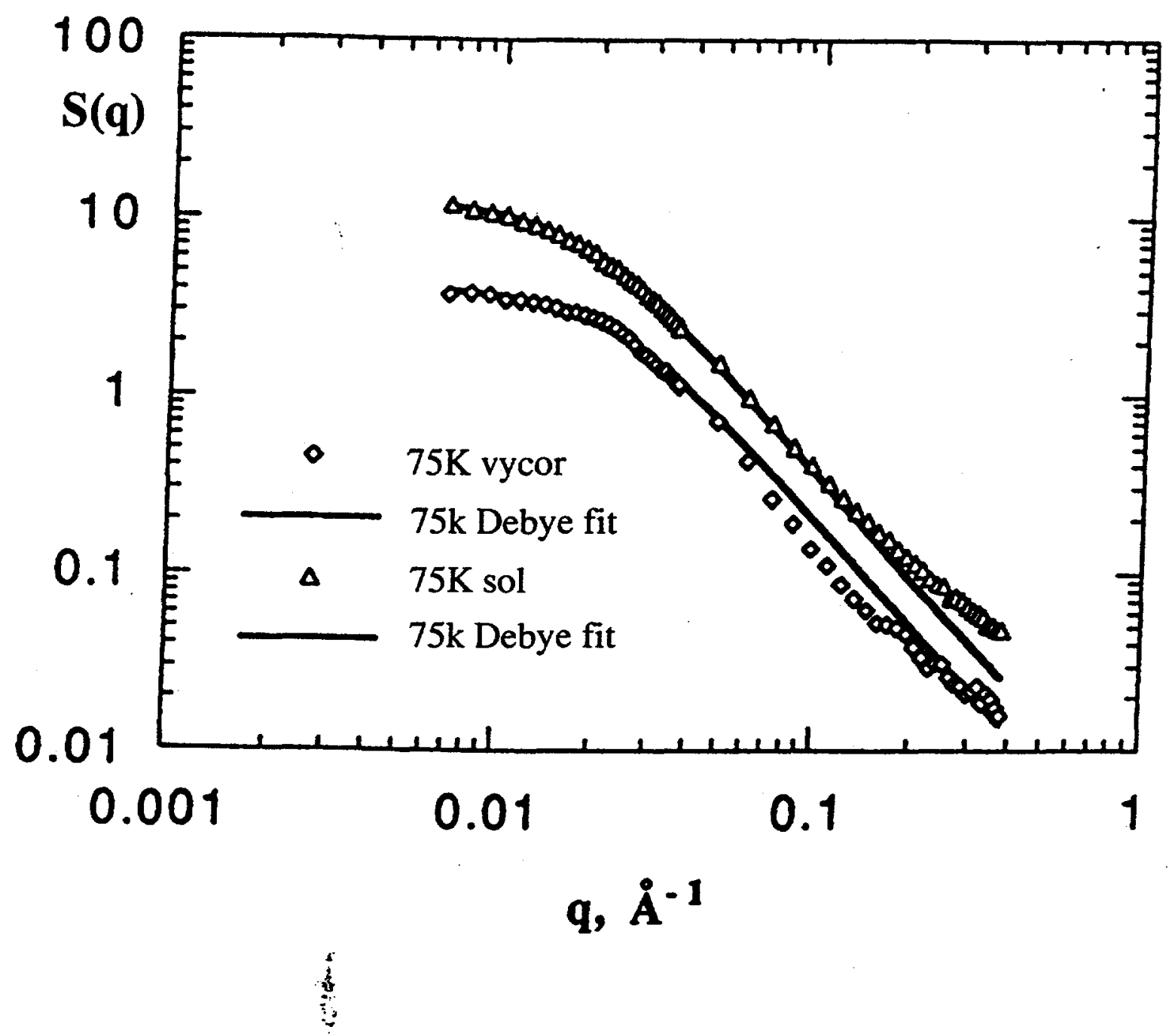

751 


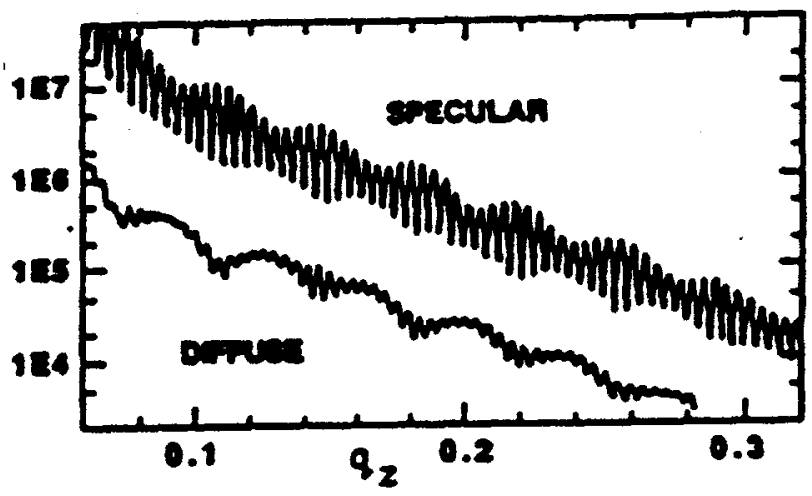

7912 